\title{
A Mathematical Model for Assessing the Impact of Intravenous Drug Misuse on the Dynamics of HIV and HCV within Correctional Institutions
}

\author{
S. Mushayabasa, Claver P. Bhunu, and Alexander G. R. Stewart \\ Department of Mathematics, University of Zimbabwe, P.O. Box MP 167, Harare, Zimbabwe \\ Correspondence should be addressed to S. Mushayabasa, steadymushaya@gmail.com
}

Received 22 October 2012; Accepted 7 November 2012

Academic Editors: M. T. Figge and W. Raffelsberger

Copyright (c) 2012 S. Mushayabasa et al. This is an open access article distributed under the Creative Commons Attribution License, which permits unrestricted use, distribution, and reproduction in any medium, provided the original work is properly cited.

Unsafe injecting practices, blood exchange, the use of nonsterile needles, and other cutting instruments for tattooing are common in correctional institutions, resulting in a number of blood transmitted infections. A mathematical model for assessing the dynamics of HCV and HIV coinfection within correctional institutions is proposed and comprehensively analyzed. The HCVonly and HIV-only submodels are first considered. Analytical expressions for the threshold parameter in each submodel and the cointeraction are derived. Global dynamics of this coinfection shows that whenever the threshold parameter for the respective submodels and the coinfection model is less than unity, then the epidemics die out, the reverse condition implies disease persistence within correctional institutions. Numerical simulations using a set of plausible parameter values are provided to support analytical findings.

\section{Introduction}

Prior studies suggests that prevalence of both human immunodeficiency virus (HIV) and hepatitis $\mathrm{C}$ virus (HCV) infections is known to be higher among incarcerated populations than the general population, since a high proportion of incarcerated individuals originate from highrisk environments and have high-risk behaviors, especially drug use [1-6]. HCV and HIV coinfections represent a public health problem of growing importance; because of similar modes of spread, many people are coinfected with $\mathrm{HCV}$ and HIV or HCV and HBV and in some cases with all three viruses at the same time [5]. In particular, HCV/HIV coinfections are common and are known as "twin epidemics" $[6,7]$. Both HIV and HIV are blood born RNA (ribonucleic acid) viruses that replicate rapidly. Unsafe injecting practices, blood exchange and the use of non-sterile needles are the most efficient means of transmitting both viruses $[6,8]$.
Correctional institutions host a disproportionately high prevalence of HCV infection and coinfections. The prevalence of $\mathrm{HCV}$ among prisoners approaches $57.5 \%$ and far exceeds that of HIV in prison $[9,10]$. Transmission of these infections is believed to be a rare consequence of blood or body fluid exposures in the prison. However, if transmission does occur, the consequences are permanent and potentially fatal [5-7]. Coinfection with the two viruses (HCV/HIV) is associated with an accelerated course of hepatitis $\mathrm{C}$ disease [5-7]. Prison populations constitute a very high-risk group; they have high levels of HCV infection and HIV or HBV coinfection $[11,12]$. HCV positive inmates are at exceptional risk for coinfection with HIV because of the association of injectable substance abuse [9-12].

Mathematical models have become invaluable management tools for epidemiologists, both shedding light on the mechanisms underlying the observed dynamics as well as making quantitative predictions on the effectiveness of different control measures. The literature and development 
of mathematical epidemiology are well documented and can be found in [13-15]. Mathematical modeling provides an alternative means to define our problems, organize our thoughts, understand our data, communicate and test our understanding, and make predictions. The deterministic compartmental model provides means of obtaining insight into on the dynamics of HIV and HCV within correctional institutions. As with most models for disease transmission and control, our model is based on the simple SIR model [16]. The main parameter of the SIR model is the basic reproduction number, $\mathcal{R}_{0}$. The basic reproductive number provides a quantitative framework to address the question of level of risk posed by a disease to a population. It allows the identification, first, of what needs to be known in order to assess risk, and, second, which of these factors are most important in determining the magnitude of risk. If this parameter $\left(\mathcal{R}_{0}\right)$ is below unity, then the disease dies out, whereas if this parameter is above unity, any small introduction of infected individuals in the population results in an oscillatory approach to an endemic equilibrium. Mathematically, there is a trivial equilibrium, known as the disease-free equilibrium, which is globally asymptotically stable whenever $\mathcal{R}_{0}<1$ [17]. A brief survey reveals that a number of statistical and mathematical studies for assessing the transmission dynamics of infectious diseases within correctional institutions have been proposed (see $[10,18-$ 24]) to mention a few. This paper seeks to use a mathematical model to asses the impact intravenous drug misuse on the dynamics of HIV and HCV within correctional institutions.

The paper is structured as follows. The HIV-HCV transmission model is formulated in the next section. The HCV submodel is presented in Section 3, followed by the HIV submodel. Section 5 presents analytical results for the HIV-HCV model formulated in Section 2. Simulation results and projection profiles of HIV-HCV model are presented in Section 6. Summary and concluding remarks round up the paper.

\section{Model Formulation}

Based on the individual's epidemiological status, the total population $N$ has been subdivided into the following classes or subgroups: susceptible nonintravenous drug users $\left(S_{n}\right)$, susceptible intravenous drug users (IDUs) $\left(S_{d}\right)$, individuals singly infected with $\mathrm{HCV}\left(I_{c}\right)$ (both infective and chronic), individuals singly infected with $\operatorname{HIV}\left(I_{h}\right)$, and dually infected individuals $\left(I_{c h}\right)$. We assume that AIDS patients dually or singly infected are not participants due to their health status. Prisoners are recruited at rate $\Lambda$. Although there is need for real demographic data for one to know the proportion of inmates recruited into each of the aforementioned epidemiological classes, we have assumed that a fraction $\pi_{0}, \pi_{1}, \pi_{2}, \pi_{3}$, and $\pi_{4}$ are recruited into $S_{n}, S_{d}, I_{c}, I_{h}$, and $I_{c h}$, respectively. Natural mortality $\mu$ is assumed to be constant in all classes. Susceptible intravenous drug users acquire either HCV or HIV but not both at rates $\lambda_{c}, \lambda_{h}$, respectively, where $\lambda_{c}=$ $\beta\left(I_{c}+\eta I_{c h}\right) / N$, and $\lambda_{h}=\theta\left(I_{h}+\eta I_{c h}\right) / N$. Parameters $\beta$ and $\theta$ denotes the probability of getting infected with either HCV or HIV, respectively. The parameter $\eta>1$ captures the assumed increased probability for individuals dually infected with HCV and HIV to infect their partners. The model takes the following form:

$$
\begin{gathered}
S_{n}^{\prime}=\Lambda \pi_{0}-(\alpha+\mu+\omega) S_{n}, \\
S_{d}^{\prime}=\Lambda \pi_{1}+\alpha S_{n}-\left(\lambda_{c}+\lambda_{h}\right) S_{d}-(\mu+\omega) S_{d}, \\
I_{c}^{\prime}=\Lambda \pi_{2}+\lambda_{c} S_{d}-\sigma \lambda_{h} I_{c}-(\mu+\omega) I_{c}, \\
I_{h}^{\prime}=\Lambda \pi_{3}+\lambda_{h} S_{d}-\sigma \lambda_{c} I_{h}-(\mu+\omega+\delta) I_{h}, \\
I_{c h}^{\prime}=\Lambda \pi_{4}+\sigma\left(\lambda_{h} I_{c}+\lambda_{c} I_{h}\right)-(\mu+\omega+\phi \delta) I_{c h} .
\end{gathered}
$$

Upon completion of their sentence, inmates are released at rate $\omega$, susceptible non-IDUs acquire IDUS behavior at rate $\alpha$, and $\sigma(\sigma>1)$ captures the assumed increased likelihood for singly infected individuals to become dually infected due to suppressed immune system. Individuals infected with HIV alone and those dually infected with HIV and HCV progress to AIDS stage at rate $\delta$, and $\phi \delta$, respectively. The modification factor $\phi>1$ captures the increased likelihood for dually infected individuals to progress to AIDS stage compared to individuals singly infected with HIV. The model flow diagram is depicted in Figure 1.

2.1. Basic Properties of the Model. In this section, we study the basic properties of the solutions of model system (1), which are essential in the proofs of stability.

Lemma 1. The equations preserve positivity of solutions.

Proof. The vector field given by the right-hand side of (1) points inward on the boundary of $\mathbb{R}_{+}^{5} \backslash\{0\}$. For example, if $S_{n}=0$, then, $S_{n}^{\prime}=\Lambda \pi_{0} \geq 0$. In an analogous manner, the same result can be shown for the other model components (variables).

Lemma 2. All solutions of system (1) are bounded.

Proof. Using system (1) we have $N^{\prime}=\Lambda-(\mu+\omega) N-\delta\left(I_{h}+\right.$ $\left.\phi I_{c h}\right) \leq \Lambda-(\mu+\omega) N$. Assume that $N(t) \leq M$ for all $t \geq 0$ where $M=(\Lambda /(\mu+\omega))+\epsilon, \epsilon>0$. Suppose the assumption is not true then there exists a $t_{1}>0$ such that

$$
\begin{gathered}
N\left(t_{1}\right)=\frac{\Lambda}{(\mu+\omega)}+\epsilon, \quad N(t)<\frac{\Lambda}{(\mu+\omega)}+\epsilon, \quad t<t_{1}, \\
N^{\prime}\left(t_{1}\right) \geq 0, \quad N^{\prime}\left(t_{1}\right) \leq \Lambda-(\mu+\omega) N\left(t_{1}\right)=-(\mu+\omega)<0,
\end{gathered}
$$

which is a contradiction meaning the assumption is true. This means $N(t) \leq M$ for all $t \geq 0$.

Therefore all feasible solutions of system (1) enter the region

$$
\Omega=\left\{\left(S_{n}, S_{d}, I_{c}, I_{h}, I_{c h}\right) \in \mathbb{R}_{+}^{5}: N \leq \frac{\Lambda}{(\mu+\omega)}\right\} .
$$

Thus, $\Omega$ is positively invariant and it is sufficient to consider solutions of system (1) in $\Omega$. Existence, uniqueness, and 


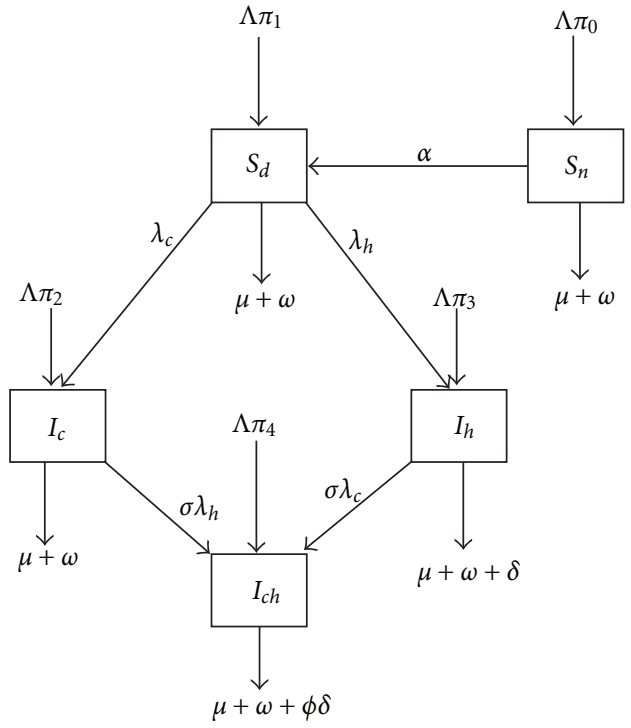

FIgURE 1: Flow diagram of the HCV transmission.

continuation results for system (1) hold in this region and all solutions of system (1) starting in $\Omega$ remain in $\Omega$ for all $t \geq 0$. All parameters and state variables for model system (1) are assumed to be nonnegative (for biological relevance) for all $t \geq 0$ since it monitors human population.

\section{HCV-Only Submodel}

Before analyzing the full model (system (1)), it is instructive to gain insights on the dynamics of the HCV-only submodel, obtained by setting $I_{h}=I_{c h}=0$, so that system (1) reduces to

$$
\begin{gathered}
S_{n}^{\prime}=\Lambda \pi_{0}-(\alpha+\mu+\omega) S_{n}, \\
S_{d}^{\prime}=\Lambda \pi_{1}+\alpha S_{n}-\left(\lambda_{c}+\mu+\omega\right) S_{d}, \\
I_{c}^{\prime}=\Lambda \pi_{2}+\lambda_{c} S_{d}-(\mu+\omega) I_{c} .
\end{gathered}
$$

For system (4), the first octant in the state space is positively invariant and attracting; that is, solutions that start where all the variables are nonnegative remain there. Thus, system (4) will be analyzed in a suitable region

$$
\Omega_{c}=\left\{\left(S_{n}, S_{d}, I_{c}\right) \in \mathbb{R}_{+}^{3}: N \leq \frac{\Lambda}{(\mu+\omega)},\right.
$$

which is positively invariant and attracting. Existence, uniqueness, and continuation results for system (4) hold in this region.

3.1. Disease-Free Equilibrium and Its Stability. System (4) has a disease-free equilibrium (DFE) given by

$$
\begin{aligned}
{\mathcal{E}_{c}^{0}}^{0} & =\left(S_{n}^{0}, S_{d}^{0}, I_{c}^{0}\right) \\
& =\left(\frac{\Lambda \pi_{0}}{(\alpha+\mu+\omega)}, \frac{\Lambda\left[\alpha \pi_{0}+\pi_{1}(\mu+\alpha+\omega)\right]}{(\mu+\omega)(\mu+\alpha+\omega)}, 0\right) .
\end{aligned}
$$

Now, we compute the basic reproductive number, $\mathcal{R}_{0}$, for system (4). Following Van Den Driessche and Watmough [17], the reproductive number for system (4) is given by

$$
\mathcal{R}_{c}=\frac{\beta\left[\alpha \pi_{0}+(\alpha+\mu+\omega) \pi_{1}\right]}{\left(\pi_{0}+\pi_{1}\right)(\mu+\omega)(\alpha+\mu+\omega)},
$$

where $\mathcal{R}_{c}$ measures the average number of new secondary cases generated by a single HCV infective individual during his/her entire infectious period when he/she is introduced into a susceptible population within a correctional setting in the absence of HCV intervention strategies. Using [17, Theorem 2], the following result is established.

Theorem 3. $\varepsilon_{c}^{0}$ is locally asymptotically stable (LAS) if $\mathcal{R}_{c}<$ 1 , and unstable if $\mathcal{R}_{c}>1$.

In order to examine the global stability of $\mathcal{E}_{c}^{0}$ we use the method proposed by Kamgang and Sallet (2008). By closely following Kamgang and Sallet (2008) [25-27], we write system (1) in the form

$$
\begin{gathered}
x_{1}^{\prime}=A_{1}(x) \cdot\left(x_{1}-x_{1}^{*}\right)+A_{12} \cdot\left(x_{2}\right), \\
x_{2}^{\prime}=A_{2}(x) x_{2},
\end{gathered}
$$

on the positively invariant set $\Omega_{c} \subset \mathbb{R}_{+}^{n_{1}+n_{2}}$. Here $x_{1}=\left(S_{n}, S_{d}\right)$ and $x_{2}=\left(I_{c}\right)$. Here $x_{1} \in \mathbb{R}_{+}^{2}$ denotes (its components) the number of uninfected individuals and $x_{2} \in \mathbb{R}_{+}^{1}$ denotes the number of infected components, $x_{1}^{*}=\mathcal{E}_{c}^{0}$. We have to prove that the following conditions are satisfied.

$\left(H_{1}\right)$ The system is defined on a positively invariant set $\Omega_{c}$ of the nonnegative orthant. The system is dissipative on $\Omega_{c}$.

$\left(H_{2}\right)$ The subsystem $x_{1}^{\prime}=A_{1} \cdot\left(x_{1}, 0\right) \cdot\left(x_{1}-x_{1}^{*}\right)$ is globally asymptotically stable at the equilibrium $x_{1}^{*}$ on the canonical projection of $\Omega_{c}$ on $\mathbb{R}_{+}^{n_{1}}$.

$\left(H_{3}\right)$ The matrix $A_{2}(x)$ is Metzler (A Metzler matrix is a matrix with off-diagonal nonnegative entries [28]) and irreducible for any given $x \in \Omega_{c}$.

$\left(H_{4}\right)$ There exists an upper-bound matrix $\bar{A}_{2}$ for $\mathbb{M}=$ $\left\{A_{2}(x) / x \in \Omega_{c}\right\}$ with the property that either $A_{2} \notin \mathbb{M}$ or if $A_{2} \notin \mathbb{M}$, (i.e., $A_{2}=\max _{\Omega_{c}} \mathbb{M}$ ), then for any $\bar{x} \in \Omega_{c}$ such that $\bar{A}_{2}=A_{2}(\bar{x}), \bar{x} \in \mathbb{R}_{+}^{n_{1}} \times\{0\}$ (i.e., the points where the maximum is realized are contained in the disease-free submanifold).

$\left(H_{5}\right) \rho\left(\bar{A}_{2}\right) \leq 0$.

If conditions $\left(H_{1}-H_{5}\right)$ are satisfied, then $\varepsilon_{0}$ is globally asymptotically stable in $\Omega_{c}$. follows:

We express the subsystem $x_{1}^{\prime}=A_{1} \cdot\left(x_{1}, 0\right) \cdot\left(x_{1}-x_{1}^{*}\right)$ as

$$
\begin{gathered}
S_{n}^{\prime}=\Lambda \pi_{0}-(\alpha+\mu+\omega) S_{n}, \\
S_{d}^{\prime}=\Lambda \pi_{1}+\alpha S_{n}-(\mu+\omega) S_{d} .
\end{gathered}
$$


System (9) is a linear system which is globally asymptotically stable at the equilibrium $\left(\Lambda \pi_{0} /(\alpha+\mu+\omega), \Lambda\left[\alpha \pi_{0}+\pi_{1}(\mu+\alpha+\right.\right.$ $\omega)] /(\mu+\omega)(\mu+\alpha+\omega))$, which corresponds to $\mathcal{E}_{c}^{0}$, satisfying conditions $\left(H_{1}\right)$ and $\left(H_{2}\right)$. Hence, matrix $A_{2}(x)$ is given by

$$
A_{2}=\left[-(\mu+\omega)\left(1-\frac{\beta S_{d}}{N(\mu+\omega)}\right)\right]
$$

Theorem 3 suggests that $\beta S_{d} / N<(\mu+\omega)$, whenever $\mathcal{R}_{c}<1$, hence matrix $A_{2}(x)$ is a Metzler matrix for any $x \in \Omega_{c}$ and this satisfies conditions $\left(H_{3}\right)$ and $\left(H_{4}\right)$. The upper bound of $A_{2}(x)$ is given by

$$
A_{2}=\left[-(\mu+\omega)\left(1-\frac{\beta\left[\alpha \pi_{0}+(\alpha+\mu+\omega) \pi_{1}\right]}{\left(\pi_{0}+\pi_{1}\right)(\mu+\omega)(\alpha+\mu+\omega)}\right)\right] .
$$

Condition $\left(H_{5}\right)$ requires that $\rho\left(A_{2}(x)\right) \leq 0$, that is,

$$
\frac{\beta\left[\alpha \pi_{0}+(\alpha+\mu+\omega) \pi_{1}\right]}{\left(\pi_{0}+\pi_{1}\right)(\mu+\omega)(\alpha+\mu+\omega)} \leq 1
$$

which is the reproductive number for system (4). Thus, $\mathcal{E}_{c}^{0}$ is globally asymptotically stable whenever $\mathcal{R}_{c} \leq 1$. We summarize the result in Theorem 4 .

Theorem 4. The disease-free equilibrium $\left(\mathcal{E}_{c}^{0}\right)$ of model system (1) is globally asymptotically stable (GAS) if $\mathcal{R}_{c} \leq 1$ and unstable if $\mathcal{R}_{c}>1$.

3.2. Permanence of the Model. We first present the following definitions that are similar to those in $[29,30]$.

Definition 5. Model system (4) is said to be uniformly persistent if there is an $\epsilon>0$ (independent of the initial data) such that every solution with positive initial conditions satisfies

$$
\begin{gathered}
\epsilon \leq \liminf _{t \rightarrow \infty} S_{n}(t), \quad \epsilon \leq \liminf _{t \rightarrow \infty} S_{d}(t), \\
\epsilon \leq \liminf _{t \rightarrow \infty} I_{c}(t) .
\end{gathered}
$$

Definition 6. Model system (4) is said to be permanent if there exists a compact region $M \subset \stackrel{\circ}{\Omega}_{c}$ (the interior of $\Omega_{c}$ ) such that every solution of system (4) with positive initial conditions will eventually enter and remain in the region $M$.

Since a test for permanence amounts to testing a suitable Lyapunov function, a suitable candidate for the model system (4) is

$$
\mathcal{L}_{c}\left(S_{n}, S_{d}, I_{c}\right)=S_{n}^{k_{1}} S_{d}^{k_{2}} I_{c}^{k_{3}},
$$

for $k_{1}, k_{2}, k_{3}>0$ [31]. This function takes value zero on $\partial \Omega_{c}$ (the boundary of $\Omega_{c}$ ) and is strictly positive in the interior of $\Omega_{c}\left(\stackrel{\circ}{\Omega}_{c}\right)$. We show that model system (4) is uniformly permanent. The function defined in (14) vanishes for any values of $\left(S_{n}, S_{d}, I_{h}\right)$ on the boundary and is strictly positive in $\stackrel{\circ}{\Omega}_{c}$, but we need to show that its derivative with respect to time for all points in $\stackrel{\circ}{\Omega}_{c}$ close to the boundary is also positive. Differentiating (14) and writing $\mathcal{L}_{c}$ for short, we obtain

$$
\begin{gathered}
\frac{\mathcal{L}_{c}^{\prime}}{\mathcal{L}_{c}}=k_{1} \frac{S_{n}^{\prime}}{S_{n}}+k_{2} \frac{S_{d}^{\prime}}{S_{d}}+k_{3} \frac{I_{c}^{\prime}}{I_{c}}:=\hbar\left(S_{n}, S_{d}, I_{c}\right), \\
\int_{0}^{T} \hbar\left(S_{n}, S_{d}, I_{c}\right) d t=\ln S_{n}^{k_{1}} S_{d}^{k_{2}} I_{c}^{k_{3}} \geq 0,
\end{gathered}
$$

for some choice of parameters such that

$$
S_{n}^{k_{1}} S_{d}^{k_{2}} I_{c}^{k_{3}}>1
$$

Condition (17) is strong because there might be some parts of $\partial \Omega$ on which $\mathcal{L}_{c}^{\prime}=0$. Therefore, we consider the time average of the derivative a condition which ensures that the boundary is a uniform repeller to orbits not starting on $\partial \Omega$. For $T$ large, the time average of the derivative is given by

$$
0<\frac{k_{1}}{T} \ln \frac{S_{n}(T)}{S_{n}(0)}+\frac{k_{2}}{T} \ln \frac{S_{d}(T)}{S_{d}(0)}+\frac{k_{3}}{T} \ln \frac{I_{c}(T)}{I_{c}(0)} .
$$

It is actually this weaker condition that makes $\mathcal{L}_{c}$ an average Lyapunov function [31]. During epidemics, $S_{n}(T)>$ $S_{n}(0), S_{d}(T)>S_{d}(0)$, consequently, the above inequality holds. Therefore, for orbits not starting on the boundary move away from it. Thus, model system (4) is permanent. Even though what orbits do away from the vicinity of the boundary of $\Omega_{c}$ is immaterial, we need to show in our case that they tend to the endemic fixed point of the system. This is accomplished by showing that $\mathcal{L}_{c}^{\prime}$ is negative semidefinite. From (15), on substituting the values of the derivatives therein, we obtain

$$
\begin{aligned}
\frac{\mathcal{L}_{c}^{\prime}}{\mathcal{L}_{c}}= & k_{1}(\mu+\alpha+\omega)\left(\frac{\pi_{0} \Lambda}{(\mu+\alpha+\omega) S_{n}}-1\right) \\
& +k_{2}\left(\lambda_{c}+\mu+\omega\right)\left(\frac{\Lambda\left[\alpha \pi_{0}+(\alpha+\mu+\omega) \pi_{1}\right]}{(\alpha+\mu+\omega)\left(\lambda_{c}+\mu+\omega\right) S_{d}}-1\right) \\
& +k_{3}(\mu+\omega)\left(\frac{\Lambda \pi_{2}+\lambda_{c} S_{d}}{(\mu+\omega) I_{c}}-1\right) .
\end{aligned}
$$

The right- hand side of the above expression is zero only at $\mathcal{E}_{c}^{*}$, the largest positive compact invariant set $\Omega_{c}$. Also,

$$
\mathcal{L}_{c}^{\prime} \geq-\left[k_{1}(\mu+\alpha+\omega)+k_{2}\left(\lambda_{c}+\mu+\omega\right)+k_{3}(\mu+\omega)\right] \mathcal{L}_{c} \leq 0,
$$

and since

$$
k_{1}(\mu+\alpha+\omega)+k_{2}\left(\lambda_{c}+\mu+\omega\right)+k_{3}(\mu+\omega):=K>0,
$$

then

$$
\frac{\mathcal{L}_{c}^{\prime}}{\mathcal{L}_{c}} \geq-K<0
$$

and consequently, $\mathcal{L}_{c}^{\prime}<0$. Hence, by LaSalle invariance principle [32] any trajectory starting in $\Omega_{c}$ moves towards the maximal invariant set $\mathcal{E}_{c}^{*}$, which is asymptotically stable in $\stackrel{\circ}{\Omega}_{c}$. 
3.3. Endemic Equilibrium and Its Stability. From model system (4), we note that $N=\Lambda /(\mu+\omega)$, and $S_{n}=\Lambda \pi_{0} /(\alpha+$ $\mu+\omega)$; hence system (4) can be rewritten as

$$
\begin{gathered}
S_{d}^{\prime}=\frac{\Lambda\left[\alpha \pi_{0}+(\alpha+\mu+\omega) \pi_{1}\right]}{(\alpha+\mu+\omega)}-\frac{\beta I_{c} S_{d}}{\Lambda /(\mu+\omega)}-(\mu+\omega) S_{d}, \\
I_{c}^{\prime}=\Lambda \pi_{2}+\frac{\beta I_{c} S_{d}}{\Lambda /(\mu+\omega)}-(\mu+\omega) I_{c} .
\end{gathered}
$$

We now study the global stability of system (23) using the Poincare-Bendixson Theorem [33]. Denote the right-hand side of (23) by $f$ and $g$ for $S_{d}$ and $I_{c}$, respectively, and choose a Dulac function as $D\left(S_{d}, I_{c}\right)=1 / S_{d} I_{c}$. Then we have

$$
\begin{aligned}
\frac{\partial(D f)}{\partial S_{d}} & +\frac{\partial(D g)}{\partial I_{c}} \\
& =-\frac{\Lambda}{S_{d} I_{c}}\left(\frac{\Lambda\left[\alpha \pi_{0}+(\alpha+\mu+\omega) \pi_{1}\right]}{(\alpha+\mu+\omega) S_{d}}+\frac{\pi_{2}}{I_{c}}\right)<0 .
\end{aligned}
$$

Thus, by Dulac's criterion, there are no periodic orbits in $\Omega_{c}$. Since $\Omega_{c}$ is positively invariant, and the endemic equilibrium exists whenever $\mathcal{R}_{c}>1$, then, it follows from the PoincareBendixson Theorem [33] that all solutions of the limiting system originating in $\Omega_{c}$ remain in $\Omega_{c}$, for all $t \geq 0$. Further, the absence of periodic orbits in $\Omega_{c}$ implies that the endemic equilibrium $\varepsilon_{c}^{*}$ of the HCV-only model is globally asymptotically stable whenever $\mathcal{R}_{c}>1$. We summarize the result in Theorem 7 .

Theorem 7. The endemic equilibrium of the HCV-only model system (4) is GAS in $\Omega_{c}$ whenever $\mathcal{R}_{c}>1$.

3.4. Sensitivity Analysis of $\mathcal{R}_{c}$. Sensitivity analysis of model parameters is very important to design and control strategies as well as a direction to future research. There are many methods [34] available for conducting sensitivity analysis such as differential analysis, response surface methodology, the Fourier amplitude sensitivity test (FAST) and other variance decomposition procedures, fast probability integration, and sampling-based procedures. In this section, sensitivity analysis based on Latin hypercube sampling (LHS) [34-39] has been performed using relevant parameters' values in Table 1. Sensitivity analysis assesses the amount and type of change inherent in the model as captured by the terms that define the reproductive number. If the reproductive number is very sensitive to a particular parameter, then a perturbation of the conditions that connect the dynamics to such a parameter may prove useful in identifying policies or intervention strategies that reduce epidemic prevalence. In this section the Partial rank correlation coefficients (PRCCs) were calculated to estimate the correlation between values of $\mathcal{R}_{c}$ and the model parameters across 1000 random draws from the empirical distribution of $\mathcal{R}_{c}$ and its associated parameters.

Figure 2 illustrates the PRCCs using $\mathcal{R}_{c}$ as an output variable. Results here suggest that HCV transmission is most sensitive to influence the magnitude of $\mathcal{R}_{c}$ than any other parameter. An increase in the magnitude of $\mathrm{HCV}$ transmission will result in an increase in the magnitude of the reproductive number, while an increase in prison release rate will lead to a decrease in $\mathcal{R}_{c}$. We now examine the dependence of the six model parameters, namely, HCV transmissibility, prison release rate, incoming susceptible IDUs, incoming susceptible non-IDUs, IDU adoption rate, and natural mortality rate using the Latin hypercube sampling technique.

A close analysis of results depicted in Figure 3 shows that HCV transmission and prison release rate are highly correlated on $\mathcal{R}_{c}$, positively and negatively, respectively. Results displayed here are in agreement with numerical findings in Figure 2 and analytical results on (25).

3.5. Sensitivity Indices of $\mathcal{R}_{c}$ Bases on Perturbation of Fixed Point Estimates. We now investigate the sensitivity of $\mathcal{R}_{c}$ with respect to each of the parameters, following Arriola and Hyman [44]. The normalized forward sensitivity index with respect to each of the parameters is presented below:

$$
\begin{aligned}
& \frac{\beta}{\mathcal{R}_{c}} \frac{\partial \mathcal{R}_{c}}{\partial \beta}=1, \\
& \frac{\omega}{\mathcal{R}_{c}} \frac{\partial \mathcal{R}_{c}}{\partial \omega}=-\frac{\omega\left(\pi_{1}(\mu+\omega)^{2}+\left(\pi_{0}+\pi_{1}\right)(\alpha+2(\mu+\omega))\right)}{(\mu+\omega)(\alpha+\mu+\omega)\left(\pi_{1}(\mu+\omega)+\left(\pi_{0}+\pi_{1}\right) \alpha\right)} \\
& =-0.996725 \text {, } \\
& \frac{\pi_{0}}{\mathcal{R}_{c}} \frac{\partial \mathcal{R}_{c}}{\partial \pi_{0}}=-\frac{\pi_{0} \pi_{1}(\mu+\omega)}{\left(\pi_{0}+\pi_{1}\right)\left(\pi_{1}(\mu+\omega)+\left(\pi_{0}+\pi_{1}\right) \alpha\right)} \\
& =-0.169235 \text {, } \\
& \frac{\pi_{1}}{\mathcal{R}_{c}} \frac{\partial \mathcal{R}_{c}}{\partial \pi_{1}}=\frac{\pi_{0} \pi_{1}(\mu+\omega)}{\left(\pi_{0}+\pi_{1}\right)\left(\pi_{1}(\mu+\omega)+\left(\pi_{0}+\pi_{1}\right) \alpha\right)} \\
& =0.169235 \text {, } \\
& \frac{\alpha}{\mathcal{R}_{c}} \frac{\partial \mathcal{R}_{c}}{\partial \alpha}=\frac{\alpha \pi_{0}(\mu+\omega)}{(\alpha+\mu+\omega)\left(\pi_{1}(\mu+\omega)+\left(\pi_{0}+\pi_{1}\right) \alpha\right)} \\
& =0.131372 \text {, } \\
& \frac{\mu}{\mathcal{R}_{c}} \frac{\partial \mathcal{R}_{c}}{\partial \mu}=-\frac{\mu\left(\pi_{1}(\mu+\omega)^{2}+\left(\pi_{0}+\pi_{1}\right)(\alpha+2(\mu+\omega))\right)}{(\mu+\omega)(\alpha+\mu+\omega)\left(\pi_{1}(\mu+\omega)+\left(\pi_{0}+\pi_{1}\right) \alpha\right)} \\
& =-0.141535 \text {. }
\end{aligned}
$$

Results in (25) suggest that HCV transmission rate $\beta$ and prison release rate $\omega$ are highly correlated with the reproductive number $\mathcal{R}_{c}$. An increase in $\beta$ will bring about an increase of the same proportion in $\mathcal{R}_{c}$ and a decrease in $\beta$ will result in a decrease in $\mathcal{R}_{c}$ with about an equivalent magnitude. Thus, a $20 \%$ increase in $\beta$ will result in $20 \%$ increase in $\mathcal{R}_{c}$. An increase in $\omega$ will lead to a decrease in $\mathcal{R}_{c}$ not by a similar magnitude, but close to it. Although $\pi_{0}$ (the proportion of incoming susceptible nonintravenous drug users) and $\pi_{1}$ 
TABLE 1: Model parameters and their interpretations.

\begin{tabular}{|c|c|c|c|c|}
\hline Parameter description & Symbol & Point estimate & Range & Source \\
\hline Proportion of prisoners recruited into $S_{n}$ & $\pi_{0}$ & 0.4 & $0-0.4$ & {$[24]$} \\
\hline Proportion of prisoners recruited into $S_{d}$ & $\pi_{1}$ & 0.3 & $0-0.3$ & {$[24]$} \\
\hline Proportion of prisoners recruited into $I_{c}$ & $\pi_{2}$ & 0.1 & $0-0.1$ & Assumed \\
\hline Proportion of prisoners recruited into $I_{h}$ & $\pi_{3}$ & 0.1 & $0-0.1$ & Assumed \\
\hline Proportion of prisoners recruited into $I_{c h}$ & $\pi_{4}$ & 0.1 & $0-0.1$ & Assumed \\
\hline Rate of progression to AIDS stage & $\delta$ & $0.125 \mathrm{yr}^{-1}$ & $0.01-0.125$ & {$[26]$} \\
\hline Recruitment rate for prisoners & $\Lambda$ & 123 per 100000 & - & {$[24,40]$} \\
\hline Modification parameter & $\sigma$ & 1.2 & $\geq 1$ & {$[24]$} \\
\hline Modification parameter & $\phi$ & 1.2 & $\geq 1$ & {$[24]$} \\
\hline Modification parameter & $\eta$ & 1.2 & $\geq 1$ & {$[24]$} \\
\hline Natural mortality rate & $\mu$ & $0.0142 \mathrm{yr}^{-1}$ & $0.01-0.02$ & {$[24]$} \\
\hline HCV transmissibility & $\beta$ & 0.1 & $0.0084-0.1$ & {$[41,42]$} \\
\hline HIV transmissibility & $\theta$ & 0.05 & $0.0084-0.1$ & {$[41,42]$} \\
\hline Behaviour change & $\alpha$ & $0.1 \mathrm{yr}^{-1}$ & $0-0.2$ & {$[24]$} \\
\hline Release rate & $\omega$ & $0.2 \mathrm{yr}^{-1}$ & $0.125-0.33$ & {$[24,43]$} \\
\hline
\end{tabular}

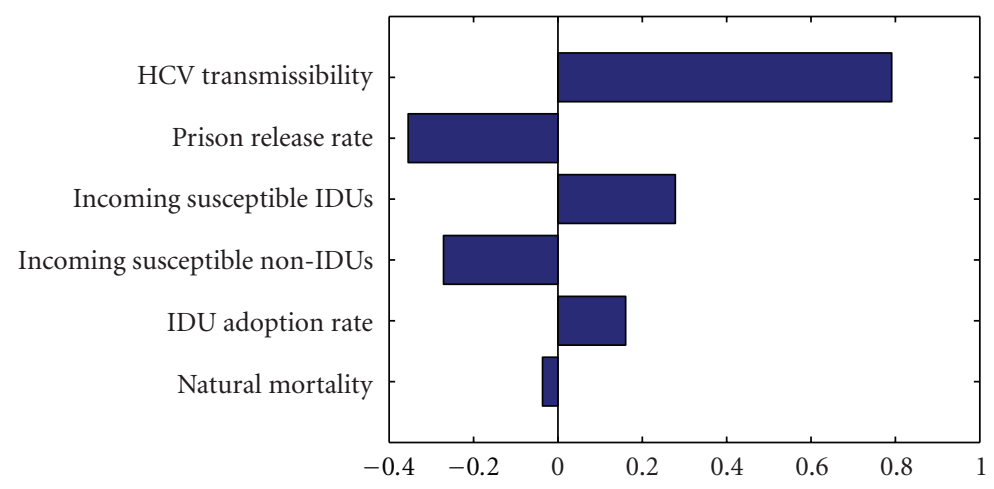

FIGURE 2: Partial rank correlation coefficients showing the effects of parameter variation on $\mathcal{R}_{c}$ using ranges in the table. Parameters with positive PRCCs will increase $\mathcal{R}_{c}$ when they are increased, whereas parameters with negative PRCCs will decrease $\mathcal{R}_{c}$ when they are increased.

(the proportion of incoming susceptible intravenous drug users) have equal influence on the magnitude of $\mathcal{R}_{c}, \pi_{0}$ will lead to a decrease in $\mathcal{R}_{c}$ and the reverse occurs for $\pi_{1}$. Results obtained here (on (25)), are in total agreement with the earlier findings in Figures 2 and 3.

\section{HIV-Only Submodel}

Consider the HIV-only submodel (obtained by setting $I_{c}=$ $\left.I_{c h}=0\right)$ in system $(1)$, so that we have

$$
\begin{gathered}
S_{n}^{\prime}=\Lambda \pi_{0}-(\alpha+\mu+\omega) S_{n}, \\
S_{d}^{\prime}=\Lambda \pi_{1}+\alpha S_{n}-\left(\lambda_{c}+\mu+\omega\right) S_{d}, \\
I_{h}^{\prime}=\Lambda \pi_{3}+\lambda_{h} S_{d}-(\mu+\omega+\delta) I_{h} .
\end{gathered}
$$

For system (26), the first octant in the state space is positively invariant and attracting; that is, solutions that start where all the variables are nonnegative remain there. Thus, system (26) will be analyzed in a suitable region

$$
\Omega_{h}=\left\{\left(S_{n}, S_{d}, I_{h}\right) \in \mathbb{R}_{+}^{3}: N \leq \frac{\Lambda}{(\mu+\omega)},\right.
$$

which is positively invariant and attracting. Existence, uniqueness, and continuation results for system (4) hold in this region.

4.1. Disease-Free Equilibrium and Its Stability. System (26) has a disease-free equilibrium (DFE) given by

$$
\begin{aligned}
\mathcal{E}_{h}^{0} & =\left(S_{n}^{0}, S_{d}^{0}, I_{h}^{0}\right) \\
& =\left(\frac{\Lambda \pi_{0}}{(\alpha+\mu+\omega)}, \frac{\Lambda\left[\alpha \pi_{0}+\pi_{1}(\mu+\alpha+\omega)\right]}{(\mu+\omega)(\mu+\alpha+\omega)}, 0\right) .
\end{aligned}
$$




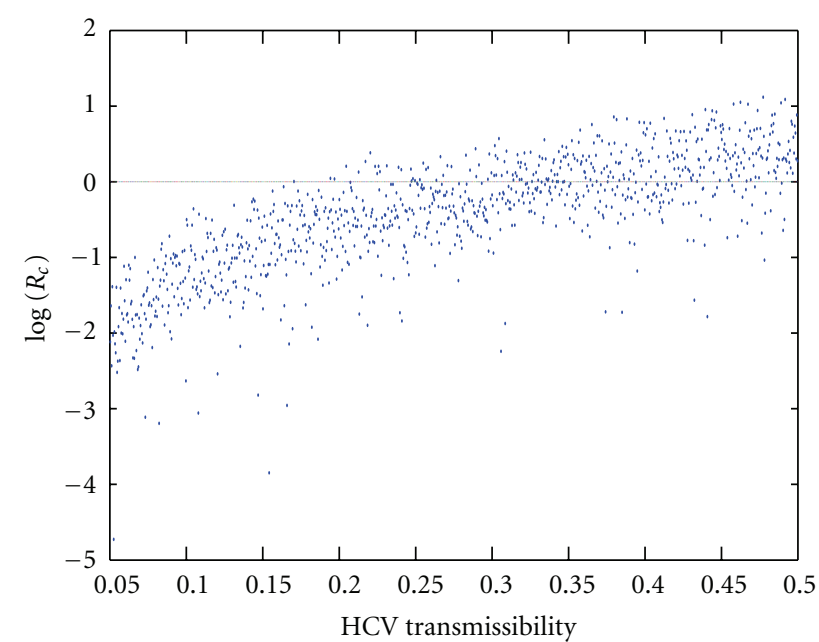

(a)

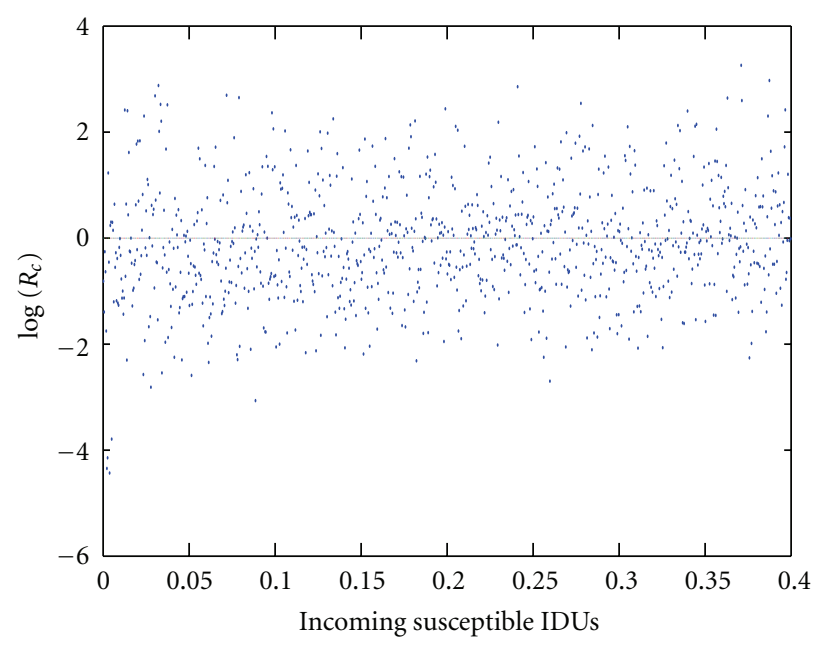

(c)

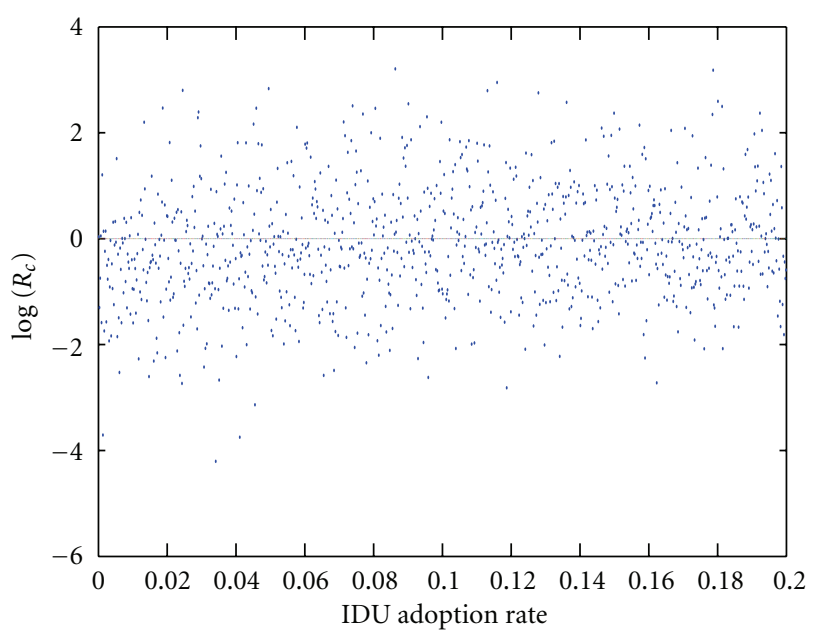

(e)

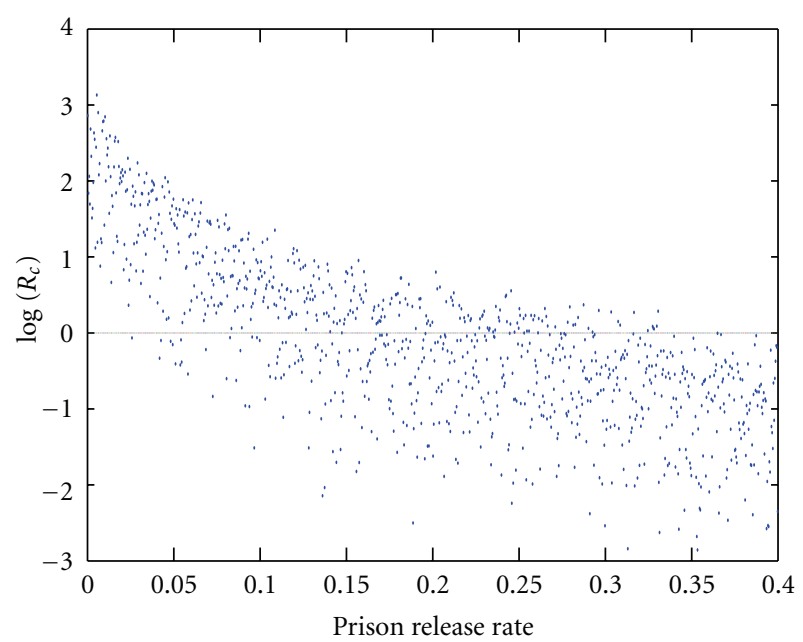

(b)

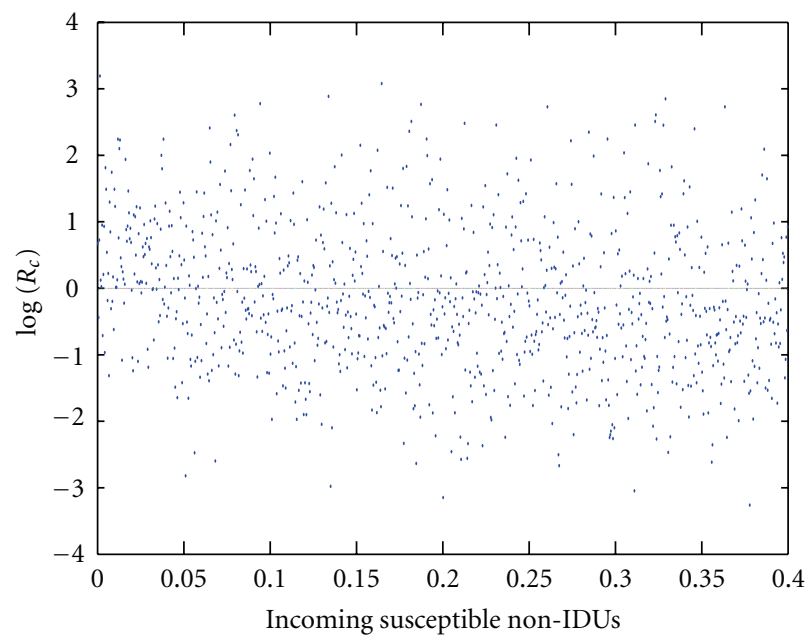

(d)

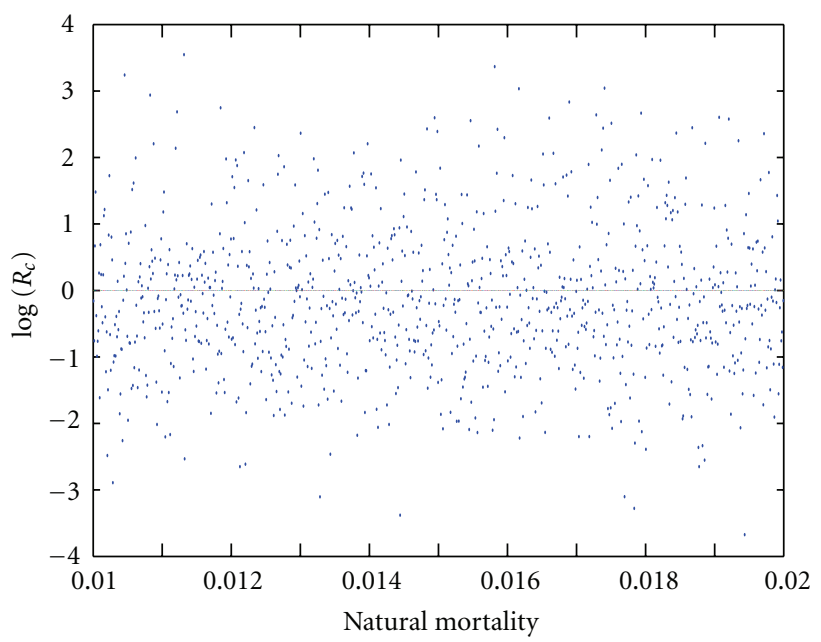

(f)

FIGURE 3: Scatter plots for the basic reproductive number $\mathcal{R}_{c}$ and six parameter values $\left(\beta, \omega, \pi_{1}, \pi_{0}, \alpha\right.$, and $\left.\mu\right)$. These results were obtained from Latin hypercube sampling using a sample size of 1000 . 
Following Van Den Driessche and Watmough [17], the reproductive number for system (26) is given by

$$
\mathcal{R}_{h}=\frac{\theta\left[\alpha \pi_{0}+(\alpha+\mu+\omega) \pi_{1}\right]}{\left(\pi_{0}+\pi_{1}\right)(\mu+\omega+\delta)(\alpha+\mu+\omega)},
$$

where $\mathcal{R}_{h}$, measures the average number of new secondary cases generated by a single HIV infective individual during his/her entire infectious period when he/she is introduced into a susceptible population within a correctional setting in the absence of HIV intervention strategies. Using [17, Theorem 2], the following result is established.

Theorem 8. The DFE $\mathcal{E}_{h}^{0}$ of system (26) is globally asymptotically stable provided $\mathcal{R}_{h} \leq 1$ and unstable if $\mathcal{R}_{h}>1$.

Proof. By closely following the approach on Section 4.1 we set

$$
x_{1}=\left(S_{n}, S_{d}\right), \quad x_{2}=\left(I_{h}\right)
$$

so that the subsystem $\dot{x}_{1}=A_{1}\left(x_{1}, 0\right) \cdot\left(x_{1}-x_{1}^{*}\right)$,

$$
\begin{gathered}
S_{n}^{\prime}=\Lambda \pi_{0}-(\alpha+\mu+\omega) S_{n}, \\
S_{d}^{\prime}=\Lambda \pi_{1}+\alpha S_{n}-(\mu+\omega) S_{d},
\end{gathered}
$$

and (31) is a linear system which is globally asymptotically stable at the equilibrium $\left(\Lambda \pi_{0} /(\alpha+\mu+\omega), \Lambda\left[\alpha \pi_{0}+\pi_{1}(\mu+\right.\right.$ $\alpha+\omega)] /(\mu+\omega)(\mu+\alpha+\omega))$ corresponding to the disease-free equilibrium $\mathcal{E}_{h}^{0}$ which satisfies conditions $\left(H_{1}\right)$ and $\left(H_{2}\right)$. The matrix $A_{2}(x)$ is given by

$$
A_{2}(x)=\left[-(\mu+\delta+\omega)\left(1-\frac{\theta S_{d}}{N(\mu+\delta+\omega)}\right)\right] .
$$

The upper bound for $x \in \Omega$ is given by

$$
\begin{aligned}
A_{2}= & {[-(\mu+\delta+\omega)} \\
& \left.\times\left(1-\frac{\theta\left[\alpha \pi_{0}+(\alpha+\mu+\omega) \pi_{1}\right]}{\left(\pi_{0}+\pi_{1}\right)(\mu+\omega+\delta)(\alpha+\mu+\omega)}\right)\right] .
\end{aligned}
$$

Thus, conditions $\left(H_{3}\right)$ and $\left(H_{4}\right)$ are satisfied. Then condition $\left(H_{5}\right)$ is equivalent to $\mathcal{R}_{h} \leq 1$,

$$
\frac{\theta\left[\alpha \pi_{0}+(\alpha+\mu+\omega) \pi_{1}\right]}{\left(\pi_{0}+\pi_{1}\right)(\mu+\omega+\delta)(\alpha+\mu+\omega)} \leq 1 .
$$

Thus, $\mathcal{E}_{h}^{0}$ is globally asymptotically stable whenever $\mathcal{R}_{h} \leq 1$.

4.2. Endemic Equilibrium and Its Stability. Following the approach on (23), system (26) can be rewritten as

$$
\begin{gathered}
S_{d}^{\prime}=\frac{\Lambda\left[\alpha \pi_{0}+(\alpha+\mu+\omega) \pi_{1}\right]}{(\alpha+\mu+\omega)}-\frac{\theta I_{h} S_{d}}{N}-(\mu+\omega) S_{d}, \\
I_{h}^{\prime}=\Lambda \pi_{2}+\frac{\theta I_{h} S_{d}}{N}-(\mu+\omega+\delta) I_{h} .
\end{gathered}
$$

In order to investigate the global stability of the endemic equilibrium, we adopt the approach by Korobeinikov (2006) [45] (and closely follow the approach in [46]). Assume that there exists $\varepsilon_{h}^{*}$ for all $S_{d}, I_{h}>\epsilon$, for some $\epsilon>0$. Let $\theta I_{h} S_{d} / N=g\left(S_{d}, I_{h}\right)$ be a positive and monotonic function, and define the following continuous function in $\mathbb{R}_{+}^{2}$ (for more details, see Korobeinikov, 2006 [45]). A function

$$
V\left(S_{d}, I_{h}\right)=S_{d}-\int_{\epsilon}^{S_{d}} \frac{g\left(S_{d}^{*}, I_{h}^{*}\right)}{g\left(\tau, I_{h}^{*}\right)} d \tau+I_{h}-\int_{\epsilon}^{I_{h}} \frac{g\left(S_{d}^{*}, I_{h}^{*}\right)}{g\left(S_{d}^{*}, \tau\right)} d \tau .
$$

If $g\left(S_{d}, I_{h}\right)$ is monotonic with respect to its variables, then the endemic state $\varepsilon_{h}^{*}$ is the only extremum and the global minimum of this function. Indeed

$$
\frac{\partial V}{\partial S_{d}}=1-\frac{g\left(S_{d}^{*}, I_{h}^{*}\right)}{g\left(S_{d}, I_{h}^{*}\right)}, \quad \frac{\partial V}{\partial I_{h}}=1-\frac{g\left(S_{d}^{*}, I_{h}^{*}\right)}{g\left(S_{d}^{*}, I_{h}\right)},
$$

grow monotonically, then the function $g\left(S_{d}, I_{h}\right)$ has only one stationary point. Further, since

$$
\begin{aligned}
& \frac{\partial^{2} V}{\partial S_{d}^{2}}=\frac{g\left(S_{d}^{*}, I_{h}^{*}\right)}{\left[g\left(S_{d}, I_{h}^{*}\right)\right]^{2}} \cdot \frac{\partial g\left(S_{d}, I_{h}^{*}\right)}{\partial S_{d}}, \\
& \frac{\partial^{2} V}{\partial I_{h}^{2}}=\frac{g\left(S_{d}^{*}, I_{h}^{*}\right)}{\left[g\left(S_{d}^{*}, I_{h}\right)\right]^{2}} \cdot \frac{\partial g\left(S_{d}^{*}, I_{h}\right)}{\partial I_{h}}
\end{aligned}
$$

are nonnegative, then the point $\varepsilon_{h}^{*}$ is a minimum. That is, $V\left(S_{d}, I_{h}\right) \geq V\left(S_{d}^{*}, I_{h}^{*}\right)$ and, hence, $V$ is a Lyapunov function, and its time derivative is given by

$$
\frac{d V}{d t}=S_{d}^{\prime}-S_{d}^{\prime}\left(\frac{g\left(S_{d}^{*}, I_{h}^{*}\right)}{g\left(S_{h}, I_{h}^{*}\right)}\right)+I_{h}^{\prime}-I_{h}^{\prime}\left(\frac{g\left(S_{d}^{*}, I_{h}^{*}\right)}{g\left(S_{d}^{*}, I_{h}\right)}\right) .
$$

Recall that

$$
\begin{gathered}
\frac{\Lambda\left[\alpha \pi_{0}+(\alpha+\mu+\omega) \pi_{1}\right]}{(\alpha+\mu+\omega)}=g\left(S_{d}^{*}, I_{h}^{*}\right)+(\mu+\omega) S_{d}^{*}, \\
\Lambda \pi_{2}=(\mu+\omega) I_{h}^{*}-g\left(S_{d}^{*}, I_{h}^{*}\right) .
\end{gathered}
$$

We have

$$
\begin{aligned}
\frac{d V}{d t}= & (\mu+\omega) S_{d}^{*}\left(1-\frac{S_{d}}{S_{d}^{*}}\right)\left(1-\frac{g\left(S_{d}^{*}, I_{h}^{*}\right)}{g\left(S_{d}, I_{h}^{*}\right)}\right) \\
& +(\mu+\omega+\delta) I_{h}^{*}\left(1-\frac{I_{h}}{I_{h}^{*}}\right)\left(1-\frac{g\left(S_{d}^{*}, I_{h}^{*}\right)}{g\left(S_{d}^{*}, I_{h}\right)}\right) \\
& +g\left(S_{d}^{*}, I_{h}^{*}\right)\left(1-\frac{g\left(S_{d}^{*}, I_{h}^{*}\right)}{g\left(S_{d}, I_{h}^{*}\right)}\right)\left(1-\frac{g\left(S_{d}^{*}, I_{h}^{*}\right)}{g\left(S_{d}, I_{h}^{*}\right)}\right) \\
& +g\left(S_{d}, I_{h}\right)\left(1-\frac{g\left(S_{d}^{*}, I_{h}^{*}\right)}{g\left(S_{d}^{*}, I_{h}\right)}\right)\left(1-\frac{g\left(S_{d}^{*}, I_{h}^{*}\right)}{g\left(S_{d}, I_{h}\right)}\right) .
\end{aligned}
$$


Since $\varepsilon_{h}^{*}>0$, the function $g\left(S_{d}, I_{h}\right)$ is concave with respect to $I_{h}$, and $\partial^{2} g\left(S_{d}, I_{h}\right) / \partial I_{h}^{2} \leq 0$, then $d V / d t \leq 0$ for all $S_{d}, I_{h}>0$. Also, the monotonicity of $g\left(S_{d}, I_{h}\right)$ with respect to $S_{d}$ and $I_{h}$ ensures that

$$
\begin{gathered}
\left(1-\frac{S_{d}}{S_{d}^{*}}\right)\left(1-\frac{g\left(S_{d}^{*}, I_{h}^{*}\right)}{g\left(S_{d}, I_{h}^{*}\right)}\right) \leq 0, \\
\left(1-\frac{I_{h}}{I_{h}^{*}}\right)\left(1-\frac{g\left(S_{d}^{*}, I_{h}^{*}\right)}{g\left(S_{d}^{*}, I_{h}\right)}\right) \leq 0, \\
\left(1-\frac{g\left(S_{d}^{*}, I_{h}^{*}\right)}{g\left(S_{d}, I_{h}^{*}\right)}\right)\left(1-\frac{g\left(S_{d}^{*}, I_{h}^{*}\right)}{g\left(S_{d}, I_{h}^{*}\right)}\right) \leq 0, \\
\left(1-\frac{g\left(S_{d}^{*}, I_{h}^{*}\right)}{g\left(S_{d}^{*}, I_{h}\right)}\right)\left(1-\frac{g\left(S_{d}^{*}, I_{h}^{*}\right)}{g\left(S_{d}, I_{h}\right)}\right) \leq 0
\end{gathered}
$$

holds for all $S_{d}, I_{h}>0$. Thus, we establish the following result.

Theorem 9. The endemic equilibrium $\varepsilon_{h}^{*}$ is globally asymptotically stable whenever conditions outlined in (42) are satisfied.

4.3. Sensitivity Analysis of $\mathcal{R}_{c}$. We now perform the sensitivity analysis of $\mathcal{R}_{h}$ and the seven model parameters, namely, HIV transmission, prison release rate, incoming susceptible IDUs, incoming susceptible non-IDUs, IDU adoption rate, progression to AIDS stage, and natural mortality rate. Results in Figure 4 suggests that prison release rate is the more sensitive to $\mathcal{R}_{h}$ than any of the aforementioned model parameters. It is also worth noting that an increase in prison release will lead to a decrease in the magnitude of $\mathcal{R}_{h}$. Although HIV transmission is not highly correlated to $\mathcal{R}_{h}$ (compared to prison release rate), its contribution on increasing the magnitude of $\mathcal{R}_{h}$ when it is increased cannot be neglected. Overall, the results depicted in Figures 2 and 4 suggest the need for HIV and HCV intervention strategies, in order to reduce the prevalence of the two diseases among prisoners.

Results depicted in Figure 5 support the earlier findings in Figure 4, that prison release rate and HIV transmission have a significant impact on influencing the magnitude of $\mathcal{R}_{h}$ negatively and positively, respectively.

4.4. Sensitivity Indices of $\mathcal{R}_{h}$ Bases on Perturbation of Fixed Point Estimates. Following Arriola and Hyman [44] we present the normalized forward sensitivity indices of $\mathcal{R}_{h}$ with respect to $\theta, \delta \mu$, and $\omega$ (note that sensitivity indices for $\mathcal{R}_{h}$ in relation to the model parameters $\pi_{0}, \pi_{1}$, and $\alpha$ yield the same results as those on (25))

$$
\begin{gathered}
\frac{\theta}{\mathcal{R}_{h}} \frac{\partial \mathcal{R}_{h}}{\partial \theta}=1, \\
\frac{\delta}{\mathcal{R}_{h}} \frac{\partial \mathcal{R}_{h}}{\partial \delta}=-\frac{\delta}{\mu+\delta+\omega}=-0.522575, \\
\frac{\mu}{\mathcal{R}_{h}} \frac{\partial \mathcal{R}_{h}}{\partial \mu} \\
=-\frac{\mu\left[(\mu+\omega)\left(\pi_{1}+2 \alpha\left(\pi_{0}+\pi_{1}\right)\right)+\alpha\left(\pi_{0} \delta+\alpha\left(\pi_{0}+\pi_{1}\right)\right)\right]}{(\mu+\alpha+\omega)(\delta+\mu+\omega)\left(\pi_{1}(\mu+\omega)+\alpha\left(\pi_{0}+\pi_{1}\right)\right)} \\
=-0.0765568, \\
\frac{\omega}{\mathcal{R}_{h}} \frac{\partial \mathcal{R}_{h}}{\partial \omega} \\
=-\frac{\omega\left[(\mu+\omega)\left(\pi_{1}+2 \alpha\left(\pi_{0}+\pi_{1}\right)\right)+\alpha\left(\pi_{0} \delta+\alpha\left(\pi_{0}+\pi_{1}\right)\right)\right]}{(\mu+\alpha+\omega)(\delta+\mu+\omega)\left(\pi_{1}(\mu+\omega)+\alpha\left(\pi_{0}+\pi_{1}\right)\right)} \\
=-0.53912 .
\end{gathered}
$$

Analytical results on (43) support the numerical findings in Figures 4 and 5.

\section{Analysis of the Full Model}

Having analysed the dynamics of the two submodels, the full HCV-HIV model is now considered (1). It is evident that system (1) has a disease-free equilibrium (DFE) given by

$$
\begin{aligned}
\mathcal{E}_{c h}^{0} & =\left(S_{n}^{0}, S_{d}^{0}, I_{c}^{0}, I_{h}^{0}, I_{c h}^{0}\right) \\
& =\left(\frac{\Lambda \pi_{0}}{(\alpha+\mu+\omega)}, \frac{\Lambda\left[\alpha \pi_{0}+\pi_{1}(\mu+\alpha+\omega)\right]}{(\mu+\omega)(\mu+\alpha+\omega)}, 0,0,0\right) .
\end{aligned}
$$

Using the next generation method, the reproductive number for system (1) is given by

$$
\begin{aligned}
\mathcal{R}_{c h}=\max \left(\frac{\beta\left[\alpha \pi_{0}+(\alpha+\mu+\omega) \pi_{1}\right]}{\left(\pi_{0}+\pi_{1}\right)(\mu+\omega)(\alpha+\mu+\omega)},\right. \\
\left.\frac{\theta\left[\alpha \pi_{0}+(\alpha+\mu+\omega) \pi_{1}\right]}{\left(\pi_{0}+\pi_{1}\right)(\mu+\omega+\delta)(\alpha+\mu+\omega)}\right) \\
=\max \left(\mathcal{R}_{c}, \mathcal{R}_{h}\right) .
\end{aligned}
$$

By closely following Kamgang and Sallet (2008) [25], and the notations of Section 4.1 we set

$$
x_{1}=\left(S_{n}, S_{n}\right), \quad x_{2}=\left(I_{c}, I_{h}, I_{c h}\right)
$$

We express the subsystem $\dot{x}_{1}=A_{1}\left(x_{1}, 0\right) \cdot\left(x_{1}-x_{1}^{*}\right)$,

$$
\begin{gathered}
S_{n}^{\prime}=\Lambda \pi_{0}-(\alpha+\mu+\omega) S_{n}, \\
S_{d}^{\prime}=\Lambda \pi_{1}+\alpha S_{n}-(\mu+\omega) S_{d},
\end{gathered}
$$




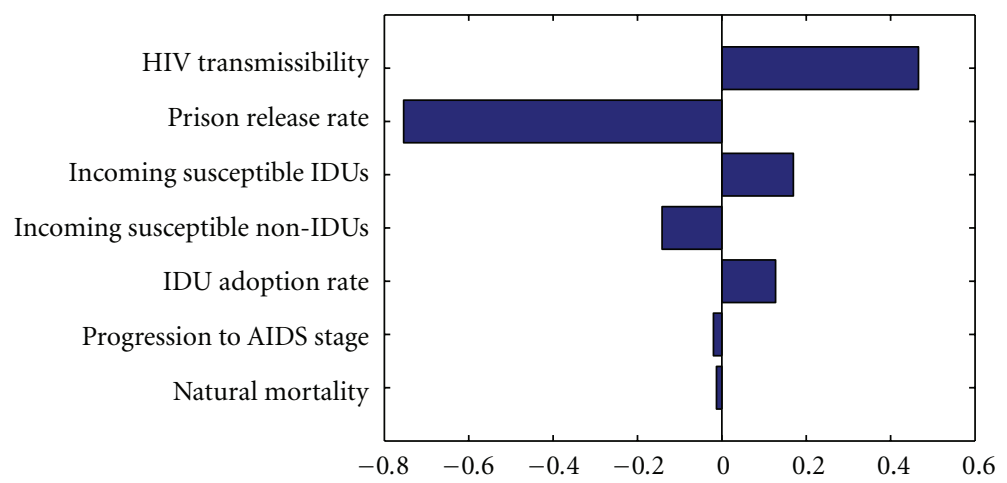

FIGURE 4: Partial rank correlation coefficients showing the effects of parameter variation on $\mathcal{R}_{h}$ using ranges in Table 1. Parameters with positive PRCCs will increase $\mathcal{R}_{h}$ when they are increased, whereas parameters with negative PRCCs will decrease $\mathcal{R}_{h}$ when they are increased.

and (47) is a linear system which is globally asymptotically stable at the equilibrium $\left(\Lambda \pi_{0} /(\alpha+\mu+\omega), \Lambda\left[\alpha \pi_{0}+\pi_{1}(\mu+\right.\right.$ $\alpha+\omega)] /(\mu+\omega)(\mu+\alpha+\omega))$ corresponding to the disease-free equilibrium $\mathcal{E}_{c h}^{0}$ which satisfies conditions $\left(H_{1}\right)$ and $\left(H_{2}\right)$. The matrix $A_{2}(x)$ is given by

$$
A_{2}(x)=\left[\begin{array}{ccc}
-\left((\mu+\omega)-\frac{\beta S_{d}}{N}\right) & 0 & \frac{\eta \beta S_{d}}{N} \\
0 & -\left((\mu+\delta+\omega)-\frac{\theta S_{d}}{N}\right) & \frac{\eta \theta S_{d}}{N} \\
0 & 0 & -(\mu+\phi \delta+\omega)
\end{array}\right]
$$

The upper bound for $x \in \Omega$ is given by

$$
A_{2}=\left[\begin{array}{ccc}
-\left((\mu+\omega)-\frac{\beta\left[\alpha \pi_{0}+(\alpha+\mu+\omega) \pi_{1}\right]}{\left(\pi_{0}+\pi_{1}\right)(\alpha+\mu+\omega)}\right) & 0 & \frac{\eta \beta\left[\alpha \pi_{0}+(\alpha+\mu+\omega) \pi_{1}\right]}{\left(\pi_{0}+\pi_{1}\right)(\alpha+\mu+\omega)} \\
0 & -\left((\mu+\delta+\omega)-\frac{\theta\left[\alpha \pi_{0}+(\alpha+\mu+\omega) \pi_{1}\right]}{\left(\pi_{0}+\pi_{1}\right)(\alpha+\mu+\omega)}\right) & \frac{\eta \theta\left[\alpha \pi_{0}+(\alpha+\mu+\omega) \pi_{1}\right]}{\left(\pi_{0}+\pi_{1}\right)(\alpha+\mu+\omega)} \\
0 & 0 & -(\mu+\phi \delta+\omega)
\end{array}\right] .
$$

Thus, conditions $\left(H_{3}\right)$ and $\left(H_{4}\right)$ are satisfied. Then condition $\left(H_{5}\right)$ is equivalent to $\mathcal{R}_{0} \leq 1$. The maximum of two quantities in (50) is the reproductive number for system (1)

$$
\begin{gathered}
\frac{\beta\left[\alpha \pi_{0}+(\alpha+\mu+\omega) \pi_{1}\right]}{\left(\pi_{0}+\pi_{1}\right)(\mu+\omega)(\alpha+\mu+\omega)} \leq 1, \\
\frac{\theta\left[\alpha \pi_{0}+(\alpha+\mu+\omega) \pi_{1}\right]}{\left(\pi_{0}+\pi_{1}\right)(\mu+\omega+\delta)(\alpha+\mu+\omega)} \leq 1 .
\end{gathered}
$$

Thus, $\mathcal{E}_{0}$ is globally asymptotically stable whenever $\mathcal{R}_{0} \leq 1$. We summarize the result in Theorem 10.

Theorem 10. The disease-free equilibrium $\left(\varepsilon_{0}\right)$ of model system (1) is globally asymptotically stable (GAS) if $\mathcal{R}_{0} \leq 1$ and unstable if $\mathcal{R}_{0}>1$.
Due to the complex nature of the endemic equilibrium for system (1), we shall use the following symmetric conditions, established from the results of Theorems 7 and 9:

(a) $\mathcal{R}_{c}<1$ (HCV dies out) and $\mathcal{R}_{h}>1$ (HIV persists),

(b) $\mathcal{R}_{h}<1$ (HIV persists) and $\mathcal{R}_{c}>1$ (HCV persists),

(c) $\mathcal{R}_{h}>1$ (HIV persists) and $\mathcal{R}_{c}>1$ (HCV persists).

The symmetric conditions above may be summarized diagrammatically as shown in Figure 6.

With the aid of the above symmetric conditions and results deduced from Theorems 7, 9, and 10, we deduce that for $\mathcal{R}_{0}>1$ the endemic equilibrium may be globally asymptotically stable. 


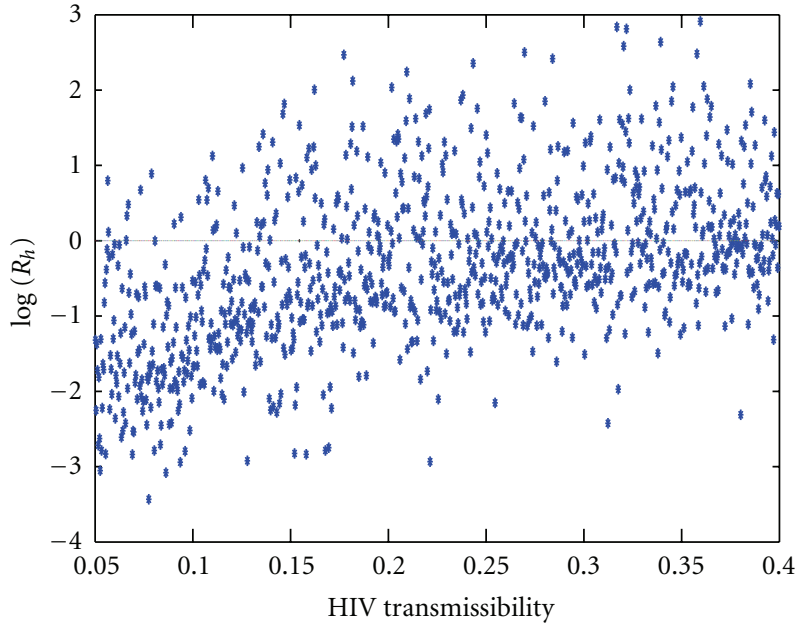

(a)

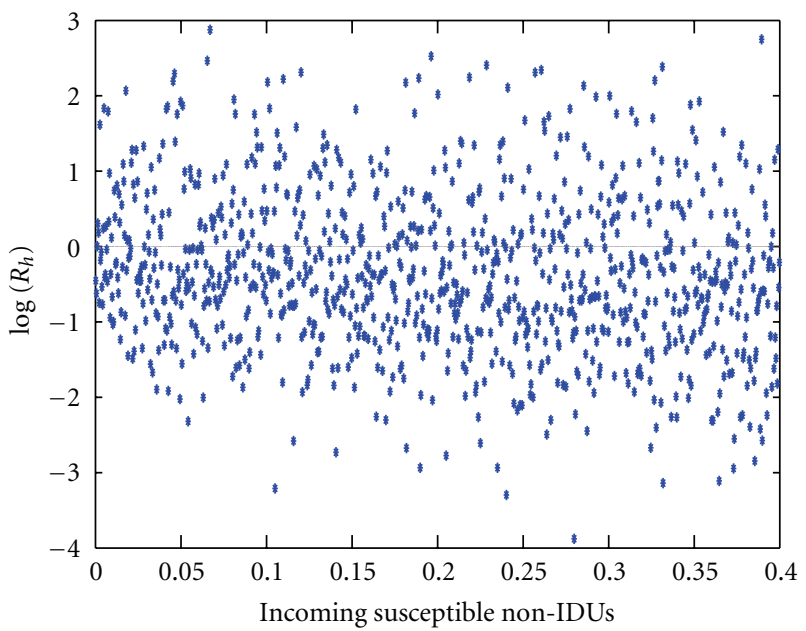

(c)

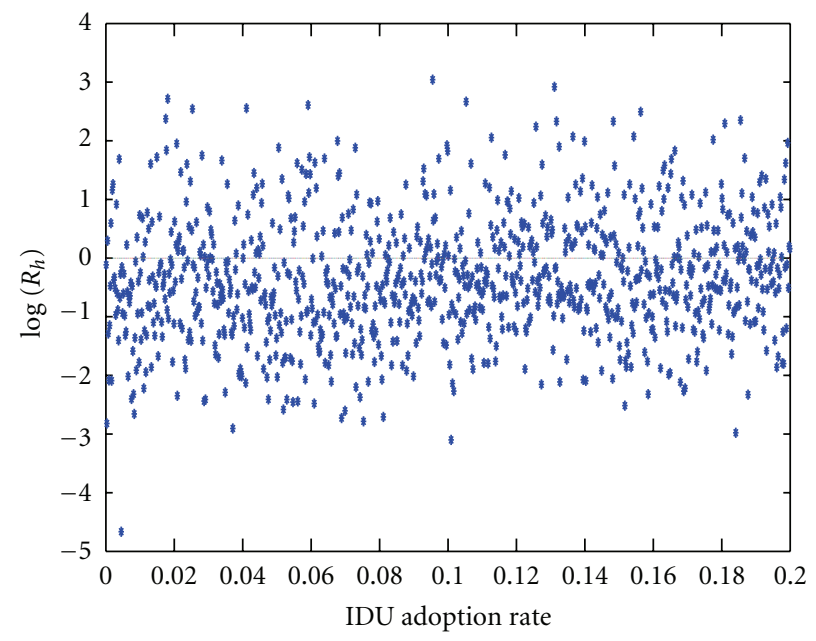

(e)

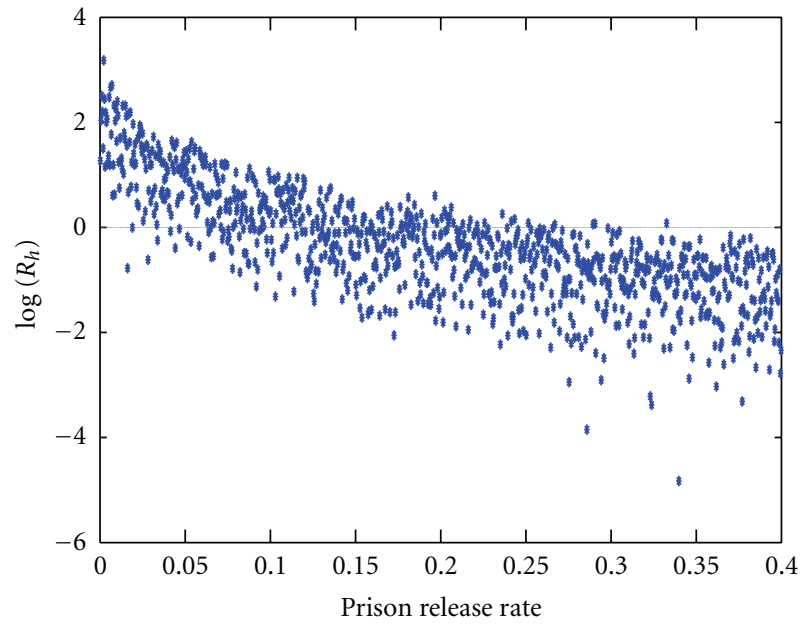

(b)



(d)

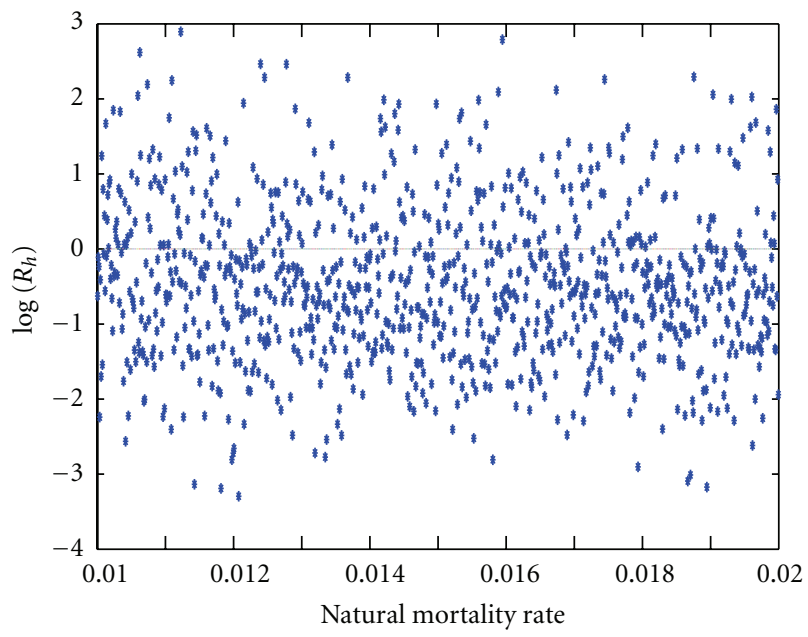

(f)

FIGURE 5: Scatter plots for the basic reproductive number $\mathcal{R}_{h}$ and seven mode parameter values $\left(\theta, \delta \omega, \pi_{1}, \pi_{0}, \alpha\right.$, and $\left.\mu\right)$. These results were obtained from Latin hypercube sampling using a sample size of 1000. 


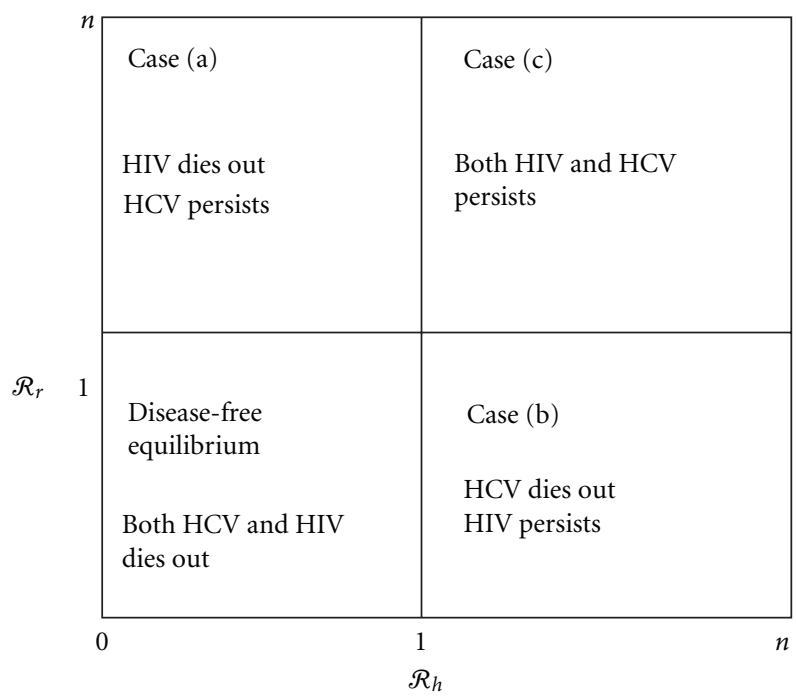

FIgURE 6: Symmetric conditions for $\mathcal{R}_{0}>1, n \in \mathcal{N},(n>1)$.

5.1. Impact of HCV on HIV Prevalence and Vice Versa. In many epidemiological models, the magnitude of the reproductive number is associated with the level of infection. The same is true for model (1). Expressions $\mathcal{R}_{c}$ and $\mathcal{R}_{h}$ can be rewritten as

$$
\begin{aligned}
\mathcal{R}_{c} & =\frac{\beta}{\left(\pi_{0}+\pi_{1}\right)(\mu+\omega)} \frac{\alpha \pi_{0}+\pi_{1}(\alpha+\mu+\omega)}{(\alpha+\mu+\omega)} \\
\Longrightarrow & \frac{\alpha \pi_{0}+\pi_{1}(\alpha+\mu+\omega)}{(\alpha+\mu+\omega)}=\frac{\left(\pi_{0}+\pi_{1}\right)(\mu+\omega) \mathcal{R}_{c}}{\beta} .
\end{aligned}
$$

Making use of the results on (51) we have

$$
\begin{aligned}
\mathcal{R}_{h} & =\frac{\theta}{\left(\pi_{0}+\pi_{1}\right)(\mu+\omega+\delta)} \frac{\alpha \pi_{0}+\pi_{1}(\alpha+\mu+\omega)}{(\alpha+\mu+\omega)} \\
\Longrightarrow & \mathcal{R}_{h}\left(\mathcal{R}_{c}\right)=\frac{(\mu+\omega) \theta \mathcal{R}_{c}}{(\mu+\omega+\delta) \beta} .
\end{aligned}
$$

Taking the partial derivative of $\mathcal{R}_{h}\left(\mathcal{R}_{c}\right)$ with respect to $\mathcal{R}_{c}$ one gets

$$
\frac{\partial \mathcal{R}_{h}\left(\mathcal{R}_{c}\right)}{\partial \mathcal{R}_{c}}=\frac{(\mu+\omega) \theta}{(\mu+\omega+\delta) \beta}>0 .
$$

Results in (53) suggest that an increase in $\mathcal{R}_{c}$ may result in an increase in $\mathcal{R}_{h}$, and this further suggests that whenever the two diseases coexist and are endemic, then they are high chances that they can influence the growth of each other. Following the above procedure in (53) it can be established that $\partial \mathcal{R}_{c}\left(\mathcal{R}_{h}\right) / \partial \mathcal{R}_{h}>0$, which again suggests that whenever the two diseases coexist they may fuel one another.

5.2. Population Level Effects. In order to support analytical findings in this study, as well as demonstrating the impact of HIV and HCV coexistence on correctional institutions, numerical simulations of the model system (1) were performed using the MATLAB ODE solver, ode45, and parameter values in Table 1 . It is worth noting that the scarcity and limited data on HIV and HCV within correctional institution limit our ability to calibrate and fit our model to the original data for future forecasts; nevertheless, we assume some of the parameters and initial conditions in the realistic range for illustrative purpose. These parsimonious assumptions reflect the lack of information currently available on the dynamics of the aforementioned epidemics with the correctional institutions. Reliable data on the two diseases (HIV and HCV) transmission within correctional institutions would enhance our understanding and aid in the possible interventions to be implemented.

Figure 7 illustrates the long term dynamics of single and dual cumulative infection cases. In Figure 7(a) we note that if $I_{c}(0)>\left(I_{h}(0)>I_{c h}(0)\right)$, then after a period of about 15 years cumulative dual cases will be more than any of the cumulative single infection cases, though cumulative $\mathrm{HCV}$ cases will remain higher than cumulative single HIV cases. In Figure 7(b) we observe that when $I_{h}(0)>\left(I_{c}(0)>I_{c h}(0)\right)$, then in a period of 5 years or less cumulative single HCV infections will be more than cumulative single HIV cases, while for a period of about 15 years cumulative dual cases will be more than either of the cumulative single infections. If $I_{h}(0)=I_{c}(0)=I_{c h}(0)$, then for the period $0-10$ years cumulative HCV single infections will be dominant followed by cumulative dual infections; however, cumulative dual infections will outnumber cumulative single HCV infections 5 years later. Overall, results in Figure 7 suggest that in the presence of HCV and HIV within correctional institutions, the first periods will be dominated by HCV cases, but time dual cases will outnumber all single infections. This clearly shows that coexistence of HCV and HIV within correctional institutions is not a pleasant news to the public health domain. 


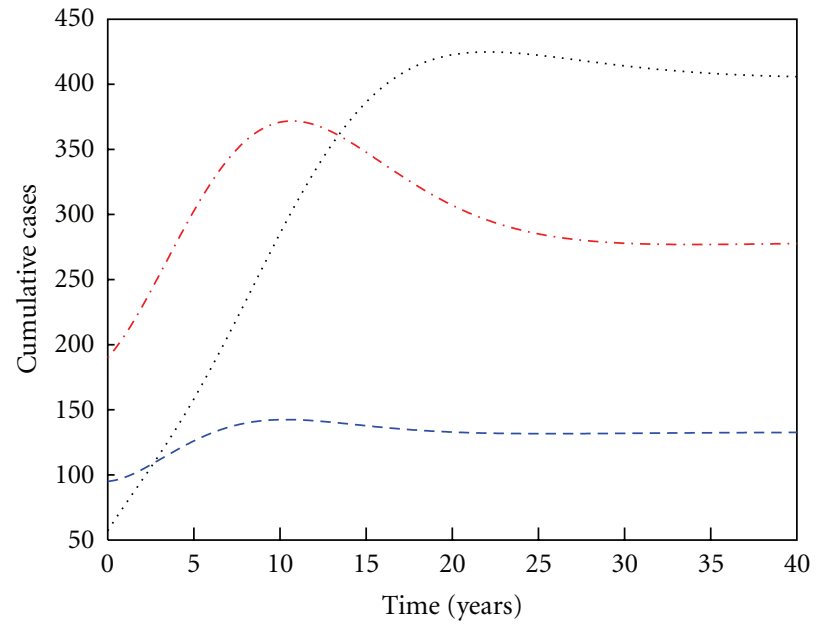

(a)



(b)

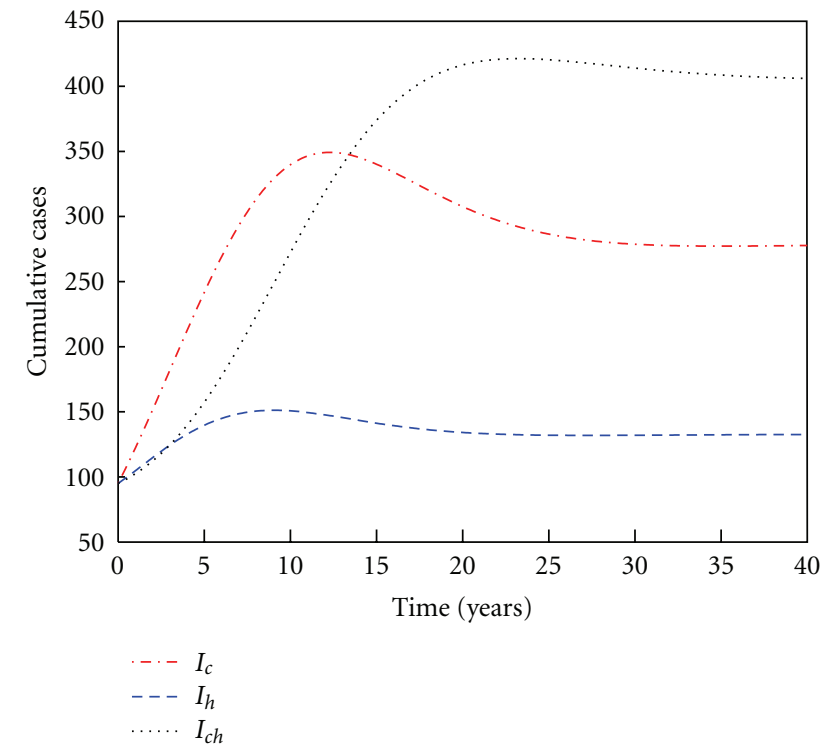

(c)

FIgURE 7: Simulations of model (1) showing the long term dynamics of $I_{c}, I_{h}$, and $I_{c h}$ for different initial conditions. The rest of the parameter values are in Table 1 . In all the three figures $S_{n}=500$ and $S_{d}=350$, while (a) $I_{c}=180, I_{h}=100$, and $I_{c h}=50 ;$ (b) $I_{c}=100, I_{h}=180$, and $I_{c h}=50$; (c) $I_{c}=100, I_{h}=100$, and $I_{c h}=100$.

Figure $8(a)$ shows that increasing $\eta$ will increase cumulative single $\mathrm{HCV}$ infections during the period 0-10 years which will also be associated with increased cumulative single HCV cases; thereafter the reverse will be true. The impact of $\eta$ on cumulative single HIV and cumulative dual cases is shown in Figures 8(b) and 8(c), respectively; here we note that an increase in $\eta$ is associated with an increase in cumulative infection cases.

The effects of increasing $\sigma$ are shown in Figure 9. Here we observe that increasing $\sigma$ may lead to a decrease in cumulative single cases, while leading to an increase in cumulative dual cases for the period $0-15$ years, and thereafter high values of $\sigma$ will be associated with low cumulative dual cases.

\section{Discussion}

HIV and hepatitis $\mathrm{C}$ virus (HCV) infections are among the most costly consequences of illicit drug use, having a high impact on individuals and on health care systems. Prison populations are considered to be at high risk for blood borne infections due to the high proportion of intravenous drug users, commercial sex workers and homeless people, highrisk sexual behaviors before and during incarceration, and tattooing among inmates [47]. A mathematical model for assessing the impact of HIV and HCV coexistence within correctional institutions has been developed and analyzed. The basic reproductive number for the model has been computed and qualitatively used to gain insights on the 


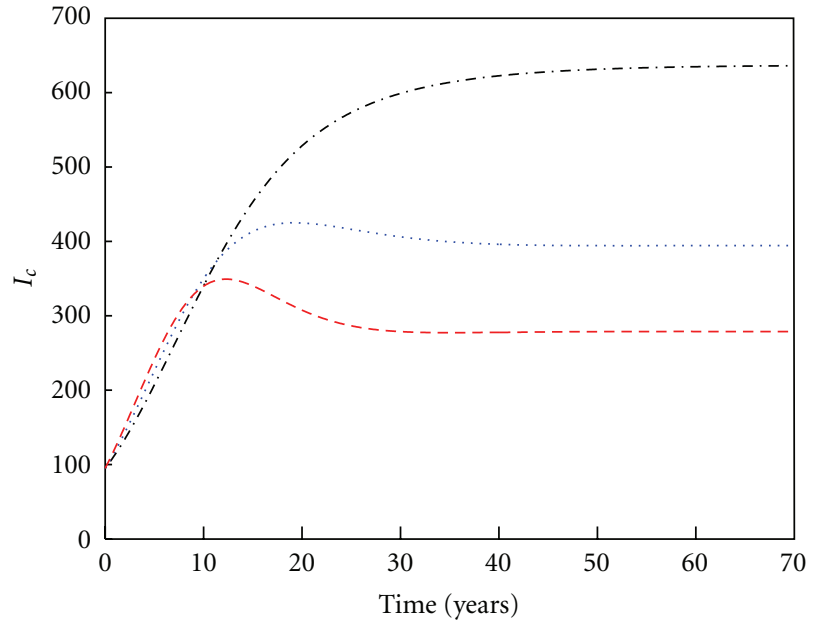

(a)

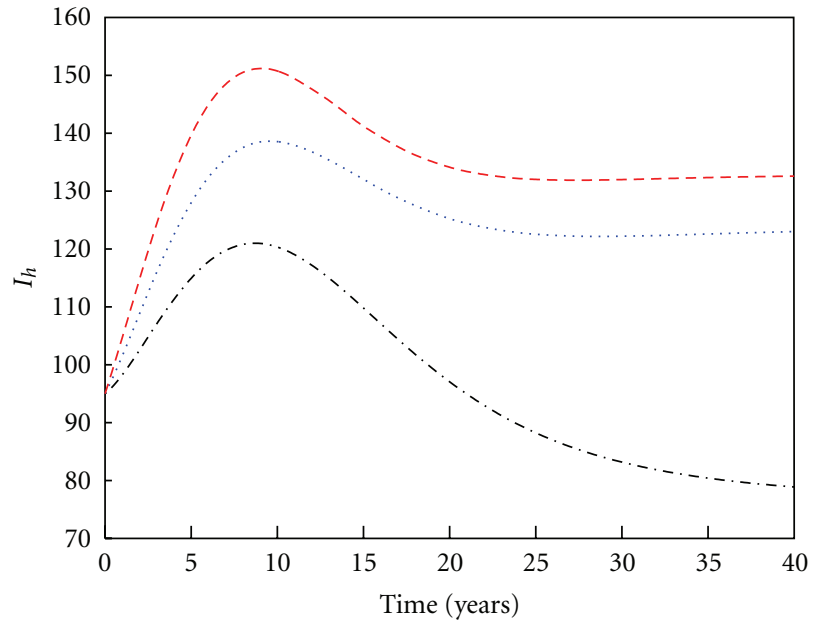

(b)

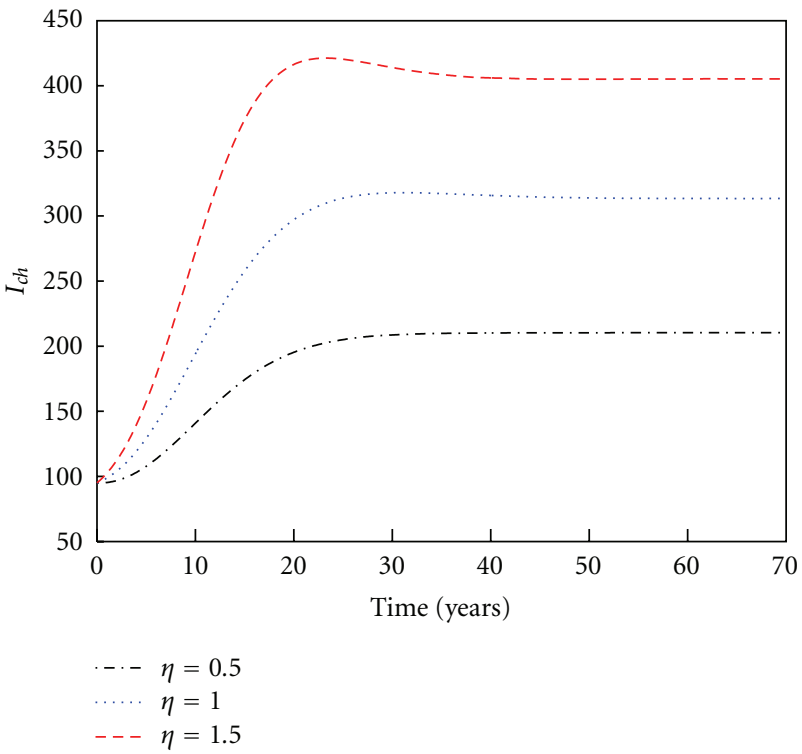

(c)

Figure 8: Simulations of model (1) showing the effects of varying the parameter $\eta$ on cumulative single HCV cases, cumulative single HIV cases, and cumulative dual cases over a period of 40 years. The following assumed initial conditions were used. $S_{n}=400, S_{d}=350$, and $I_{c}=I_{h}=I_{c h}=100$, and parameter values used are as in Table 1.

long term dynamics on cumulative single and dual cases when the two diseases coexist with correctional institution. Sensitivity analysis on the associated reproductive number has been carried out, and the influence of each of the parameters which define it (the reproductive number) has been clearly illustrated. Analysis of the two submodels (the HIV submodel and HCV submodel) suggest that each submodel has globally asymptotically disease-free equilibrium whenever the associated reproductive number is less than unity, and a globally asymptotically stable endemic when the associated reproductive number is greater than unity.
Comprehensive numerical simulations performed using the MATLAB ODE solver, ode45, have been provided to support analytical results. At its best the study suggests that whenever HIV and HCV coexist within correctional institutions, then the long-term cumulative cases will be dominated by dual infections, followed by cumulative single HCV cases, and this clearly suggests the need for resources and attention in order to control the aforementioned epidemics since the consequences will also be felt by the population outside the correction institutions. 


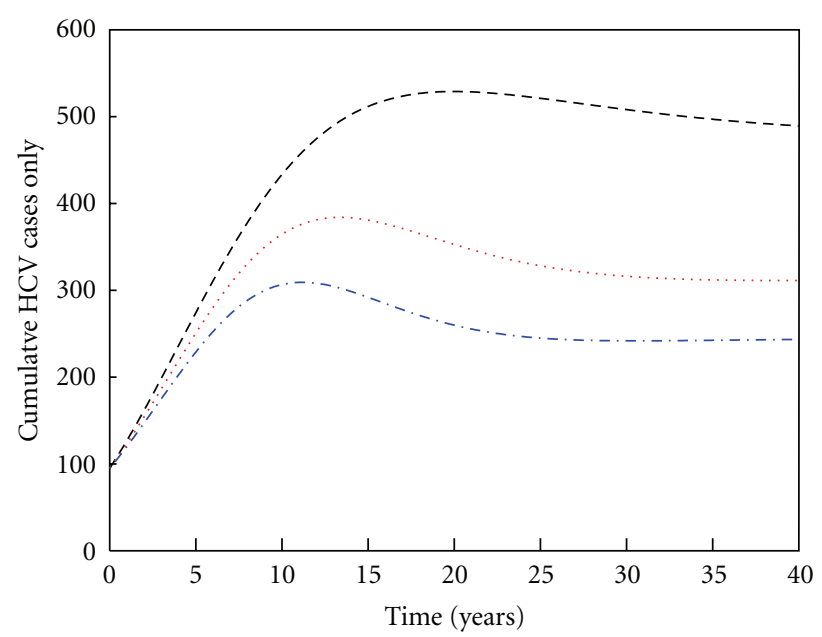

(a)

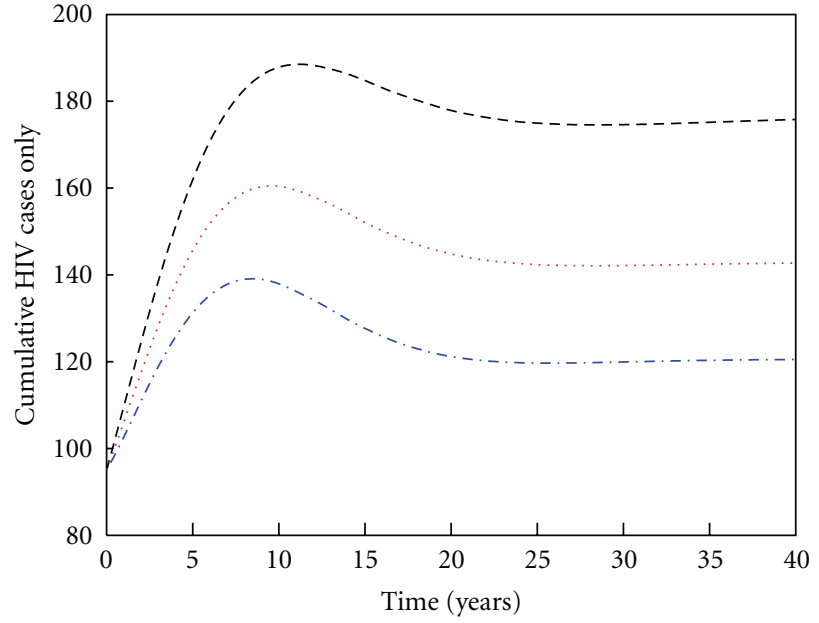

(b)

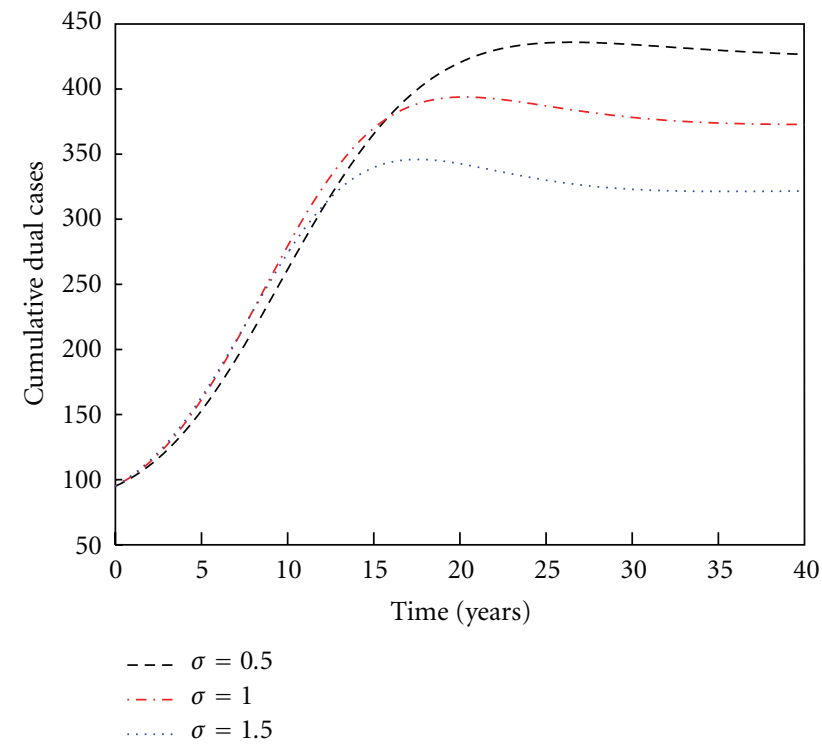

(c)

FIgURE 9: Simulations of model (1) showing the effects of varying the parameter $\sigma$ on cumulative single HCV cases, cumulative single HIV cases, and cumulative dual cases over a period of 40 years. The following assumed initial conditions were used. $S_{n}=400, S_{d}=350$, and $I_{c}=I_{h}=I_{c h}=100$, and parameter values used are as in Table 1 .

\section{Acknowledgments}

The author are grateful to the anonymous referee and the handling editor for their valuable comments and suggestions.

\section{References}

[1] Canadian HIV/AIDS Legal Network, "HIV and hepatitis C in prison," 2008

[2] K. V. Dumchev, R. Soldyshev, H. Z. Qian et al., "HIV and hepatitis $\mathrm{C}$ virus infections among hanka injection drug users in central Ukraine: a cross-sectional survey," Harm Reduction Journal, vol. 6, article 23, 2009.
[3] C. Aceijas and T. Rhodes, "Global estimates of prevalence of HCV infection among injecting drug users," International Journal of Drug Policy, vol. 18, no. 5, pp. 352-358, 2007.

[4] E. F. White, R. S. Garfein, K. C. Brouwer et al., "Prevalence of hepatitis $\mathrm{C}$ virus and HIV infection among injection drug users in two Mexican cities bordering the U.S.," Salud Publica de Mexico, vol. 49, no. 3, pp. 165-172, 2007.

[5] K. A. Hennessey, A. A. Kim, V. Griffin, N. T. Collins, C. M. Weinbaum, and K. Sabin, "Prevalence of infection with hepatitis B and $\mathrm{C}$ viruses and co-infection with HIV in three jails: a case for viral hepatitis prevention in jails in the United States," Journal of Urban Health, vol. 86, no. 1, pp. 93-105, 2009.

[6] E. Pontali and F. Ferrari, "Prevalence of Hepatitis B virus and/or Hepatitis C virus co-infections in prisoners infected 
with the Human Immunodeficiency Virus," International Journal of Prisoner Health, vol. 4, no. 2, pp. 77-82, 2008.

[7] G. E. Macalino, D. Dhawan, and J. D. Rich, "A missed opportunity: hepatitis C screening of prisoners," American Journal of Public Health, vol. 95, no. 10, pp. 1739-1740, 2005.

[8] M. F. Vescio, B. Longo, S. Babudieri et al., "Correlates of hepatitis $\mathrm{C}$ virus seropositivity in prison inmates: a metaanalysis," Journal of Epidemiology and Community Health, vol. 62, no. 4, pp. 305-313, 2008.

[9] M. E. Hellard, J. S. Hocking, and N. Crofts, "The prevalence and the risk behaviours associated with the transmission of hepatitis C virus in Australian correctional facilities," Epidemiology and Infection, vol. 132, no. 3, pp. 409-415, 2004.

[10] M. S. E. Anwar, M. Nafees, and U. Nabi, "Sero-prevalence of HCV and associated infections with HIV and HBV among prisoners in Lahore," Biomedica, vol. 27, no. 2, pp. 119-1122, 2011.

[11] A. A. Adjei, H. B. Armah, F. Gbagbo et al., "Correlates of hepatitis $\mathrm{C}$ virus infection among incarcerated Ghanaians: a national multicentre study," Journal of Medical Microbiology, vol. 56, no. 3, pp. 391-397, 2007.

[12] T. Guimarães, C. F. Granato, D. Varella, M. L. Ferraz, A. Castelo, and E. G. Kallás, "High prevalence of hepatitis C infection in a Brazilian prison: identification of risk factors for infection," The Brazilian Journal of Infectious Diseases, vol. 5, no. 3, pp. 111-118, 2001.

[13] R. M. M. Anderson, Infectious Diseases of Humans, Dynamics and Control, Oxford University Press, 1991.

[14] N. Bailey, The Mathematical Theory of Infectious Diseases, Charles Griffin, 1975.

[15] F. Brauer and C. Castillo-Chávez, Mathematical Models in Population Biology and Epidemiology, Springer, 2001.

[16] H. W. Hethcote, "Mathematics of infectious diseases," SIAM Review, vol. 42, no. 4, pp. 599-653, 2000.

[17] P. Van Den Driessche and J. Watmough, "Reproduction numbers and sub-threshold endemic equilibria for compartmental models of disease transmission," Mathematical Biosciences, vol. 180, pp. 29-48, 2002.

[18] D. J. Daley and J. Gani, Epidemic Modelling: An Introduction, vol. 15, Cambridge University Press, Cambridge, UK, 1999.

[19] S. Mushayabasa and C. P. Bhunu, "Modeling HIV transmission dynamics among male prisoners in Sub-Saharan Africa," International Journal of Applied Mathematics, vol. 41, no. 1, pp. 62-67, 2011.

[20] E. Kajita, J. T. Okano, E. N. Bodine, S. P. Layne, and S. Blower, "Modelling an outbreak of an emerging pathogen," Nature Reviews Microbiology, vol. 5, no. 9, pp. 700-709, 2007.

[21] A. A. Adjei, H. B. Armah, F. Gbagbo et al., "Correlates of hepatitis $\mathrm{C}$ virus infection among incarcerated Ghanaians: a national multicentre study," Journal of Medical Microbiology, vol. 56, no. 3, pp. 391-397, 2007.

[22] E. Gough, M. C. Kempf, L. Graham et al., "HIV and Hepatitis $\mathrm{B}$ and $\mathrm{C}$ incidence rates in US correctional populations and high risk groups: a systematic review and meta-analysis," $B M C$ Public Health, vol. 10, article 777, 2010.

[23] B. H. McGovern, A. Wurcel, A. Y. Kim et al., "Acute hepatitis C virus infection in incarcerated injection drug users," Clinical Infectious Diseases, vol. 42, no. 12, pp. 1663-1670, 2006.

[24] S. Mushayabasa, C. P. Bhunu, and R. J. Smith, "Assessing the impact of educational campaigns on controlling HCV among women in prison settings," Communications in Nonlinear Science and Numerical Simulation, vol. 17, no. 4, pp. 1714$1724,2012$.
[25] J. C. Kamgang and G. Sallet, "Computation of threshold conditions for epidemiological models and global stability of the disease-free equilibrium (DFE)," Mathematical Biosciences, vol. 213, no. 1, pp. 1-12, 2008.

[26] S. Mushayabasa and C. P. Bhunu, "Is HIV infection associated with an increased risk for cholera? Insights from a mathematical model," Biosystems, vol. 109, pp. 203-213, 2012.

[27] S. Mushayabasa CP Bhunu, "Assessing the impact of increasing antimicrobial resistance of Vibro cholerae on the future trends of cholera epidemic," ISRN Biomathematics. In press.

[28] A. Berman and R. J. Plemmons, "Nonnegative matrice in the mathematical sciences," SIAM Review, vol. 35, pp. 43-79, 1993.

[29] Y. Kuang, Delay Differential Equations with Applications in Population Dynamics, vol. 191, Academic Press, Boston, Mass, USA, 1993.

[30] Y. Xiao and L. Chen, "Modeling and analysis of a predatorprey model with disease in the prey," Mathematical Biosciences, vol. 171, no. 1, pp. 59-82, 2001.

[31] J. Hofbauer and K. Sigmund, The Theory of Evolution and Dynamical Systems, vol. 7, Cambridge University Press, Cambridge, UK, 1988.

[32] J. P. LaSalle, The Stability of Dynamical Systems, vol. 25 of CBMS-NSF Regional Conference Series in Applied 398 Mathematics, Society for Industrial and Applied Mathematics, Philadelphia, Pa, USA, 1976.

[33] L. Perko, Differential Equations and Dynamical Systems, vol. 7 of Text in Applied Mathematics, Springer, Berlin, Germany, 2000.

[34] J. C. Helton, "Uncertainty and sensitivity analysis techniques for use in performance assessment for radioactive waste disposal," Reliability Engineering and System Safety, vol. 42, no. 2-3, pp. 327-367, 1993.

[35] A. Saltelli, Sensitivity Analysis, John Wiley \& Sons, Chichester, UK, 2000.

[36] A. Mubayi, P. Greenwood, X. Wang et al., "Types of drinkers and drinking settings: an application of a mathematical model," Addiction, vol. 106, no. 4, pp. 749-758, 2011.

[37] M. Samsuzzoha and S. M. Lucy, "Uncertainty and sensitivity analysis of the basic reproductive number of a vaccinated epidemic model of influenza," Applied Mathematical Modelling, vol. 145, no. 12, pp. 1127-1137, 1997.

[38] C. P. Bhunu, S. Mushayabasa, and R. J. Smith, "Assessing the effects of poverty in tuberculosis transmission dynamics," Applied Mathematical Modelling, vol. 36, no. 9, pp. 4173-4185, 2012.

[39] S. M. Blower and H. Dowlatabadi, "Sensitivity and uncertainty analysis of complex models of disease transmission: an HIV model, as an example," International Statistical Review, vol. 2, pp. 229-243, 1994.

[40] Incarceration is not an equal opportunity punishment, http://www.prisonpolicy.org.

[41] C. P. Bhunu and S. Mushayabasa, "Assessing the effects of intravenous drug use on hepatitis C transmission dynamics," Journal of Biological Systems, vol. 19, no. 3, pp. 447-460, 2011.

[42] P. Vickerman, M. Hickman, and A. Judd, "Modelling the impact on Hepatitis C transmission of reducing syringe sharing: London case study," International Journal of Epidemiology, vol. 36, no. 2, pp. 396-405, 2007.

[43] K. Dolan, A. Wodak, and R. Penny, "AIDS behind bars: preventing HIV spread among incarcerated drug injectors," AIDS, vol. 9, no. 8, pp. 825-832, 1995.

[44] L. Arriola and J. Hyman, Lecture notes, forward and adjoint sensitivity analysis: with applications in Dynamical Systems, 
Linear Algebra and Optimisation Mathematical and Theoretical Biology 414 Institute, Summer, 2005.

[45] A. Korobeinikov, "Lyapunov functions and global stability for SIR and SIRS epidemiological models with non-linear transmission," Bulletin of Mathematical Biology, vol. 68, no. 3, pp. 615-626, 2006.

[46] S. Mushayabasa, C. P. Bhunu, C. Webb, and M. Dhlamini, "A mathematical model for assessing the impact of poverty on yaws eradication," Applied Mathematical Modelling, vol. 36, no. 4, pp. 1653-1667, 2012.

[47] A. H. M. Alizadeh, S. M. Alavian, K. Jafari, and N. Yazdi, "Prevalence of hepatitis $\mathrm{C}$ virus infection and its related risk factors in drug abuser prisoners in Hamedan-Iran," World Journal of Gastroenterology, vol. 11, no. 26, pp. 4085-4089, 2005. 


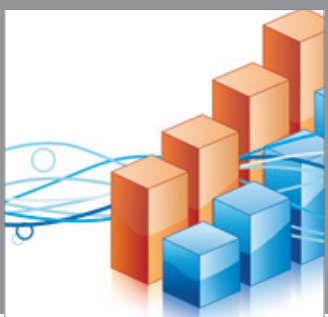

Advances in

Operations Research

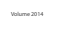

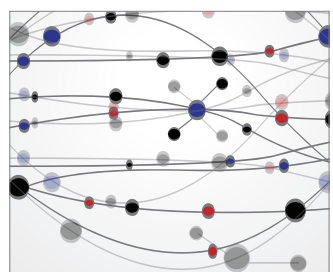

\section{The Scientific} World Journal
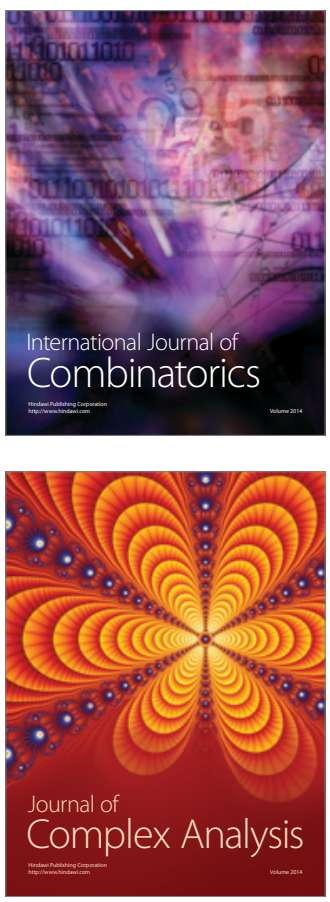

International Journal of

Mathematics and

Mathematical

Sciences
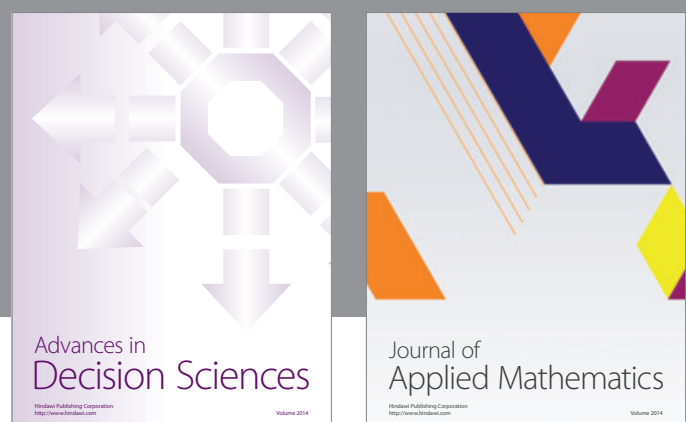

Journal of

Applied Mathematics
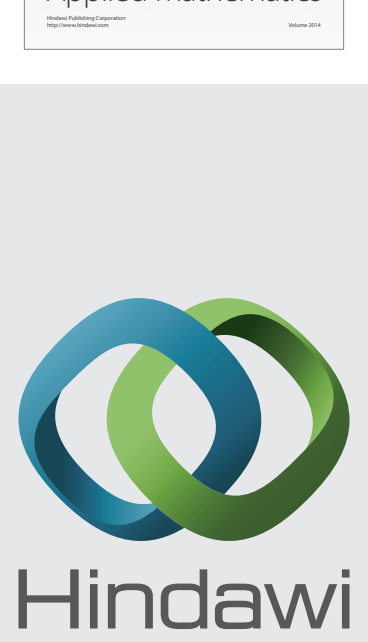

Submit your manuscripts at http://www.hindawi.com


Mathematical Problems in Engineering


Journal of

Function Spaces
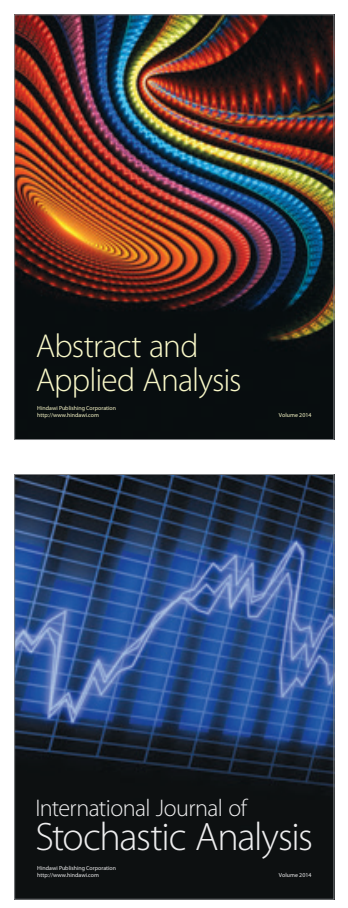

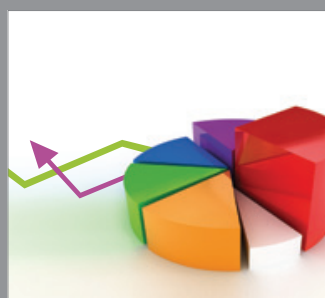

ournal of

Probability and Statistics

Promensencen
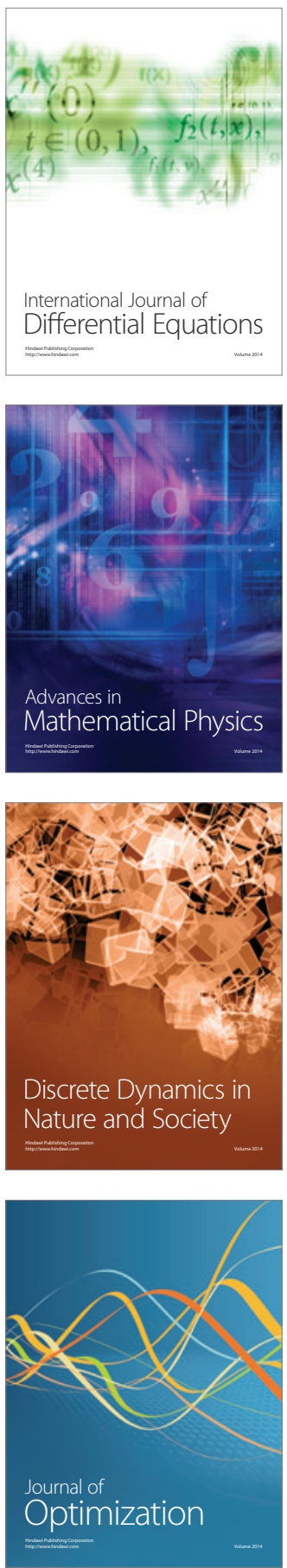